University of Redlands

\title{
Quantifying Wetland Habitat Interspersion Using Drone Imagery
}

\author{
A Major Individual Project submitted in partial satisfaction of the requirements \\ for the degree of Master of Science in Geographic Information Systems \\ by \\ Ava Gotthard \\ Ruijin Ma, Ph.D., Committee Chair \\ Mark Kumler, Ph.D.
}

August 2020 
Quantifying Wetland Habitat Interspersion Using Drone Imagery

Copyright @ 2020

by

Ava Gotthard 
The report of Ava Gotthard is approved.

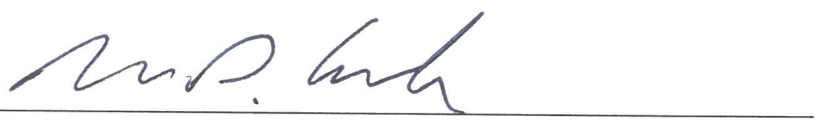
Mark Kumler, Ph.D.

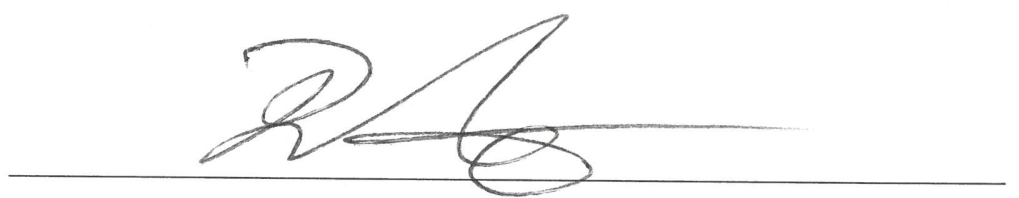

Ruijin Ma, Ph.D., Committee Chair

August 2020 


\section{Acknowledgements}

I would like to thank my Advisor and the University of Redlands for their guidance and expertise. I would also like to thank my Liaisons at the National Audubon Society for their patience and assistance in answering my questions and helping to navigate during this strange pandemic period. My family's support and encouragement cannot be overlooked either. This program has allowed me to grow in new and unexpected ways. I would not have been able to get to this point without the support of all these people including my fellow Cohort 36 members. 


\begin{abstract}
Quantifying Wetland Habitat Interspersion by Using Drone Imagery

by

Ava Gotthard

Wetland bird species are affected negatively by increases in invasive plant species in their habitat. The National Audubon Society compiled Wigwam Bay State Wildlife Area marsh drone imagery for two years and needed a marsh bird habitat model with interspersion rates at selected sites. The interspersion and juxtaposition index (IJI) method from the landscapemetrics library was used to establish a baseline and evaluate habitat change between the summers of 2018 and 2019. The landscape level metric results showed small increases in interspersion for eight of the nine study areas. However, at the class level, interspersion for Emergent Vegetation decreased in all but one study area, suggesting that despite the two-year positive trend at the landscape level,
\end{abstract} invasive plants increased from 2018 to 2019. 


\section{Table of Contents}

Chapter 1 - Introduction .........................................................................................................1

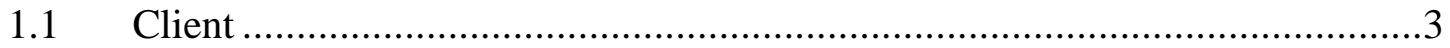

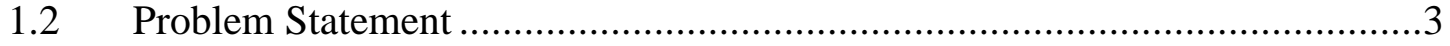

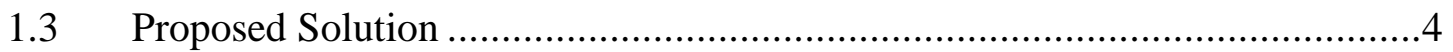

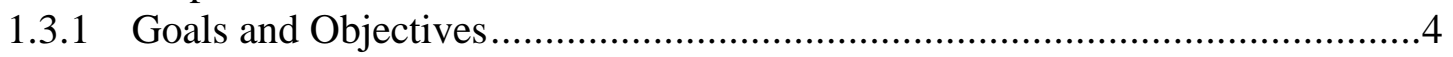

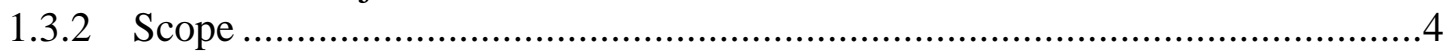

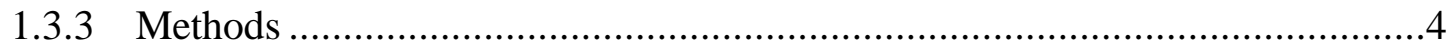

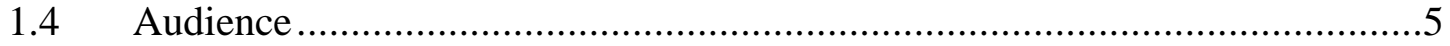

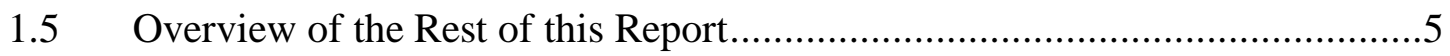

Chapter 2 - Background and Literature Review.............................................................6

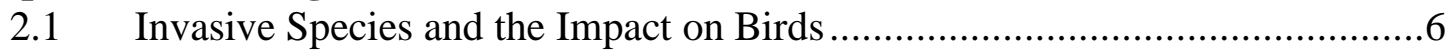

$2.2 \quad$ Species Interspersion and Calculation..........................................................

2.3 Land Cover and Species Mapping with Remote Sensing ………....................

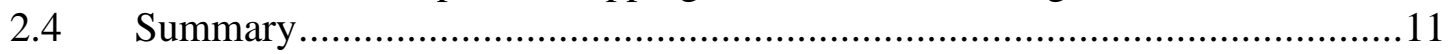

Chapter 3 - Systems Analysis and Design ..................................................................13

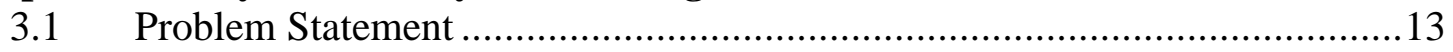

3.2 Requirements Analysis .............................................................................13

3.3 System Design and Imagery Related Decisions .............................................14

$3.4 \quad$ Project Plan ............................................................................................

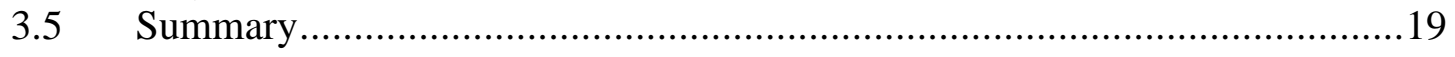

Chapter 4 - Database Design ....................................................................................21

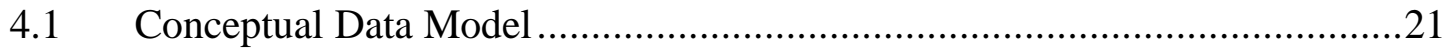

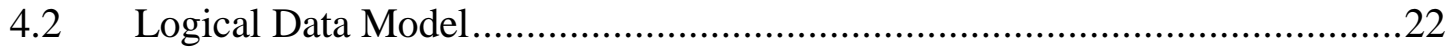

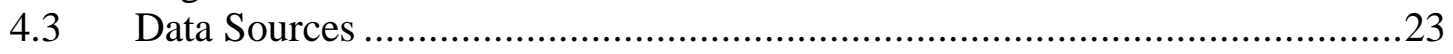

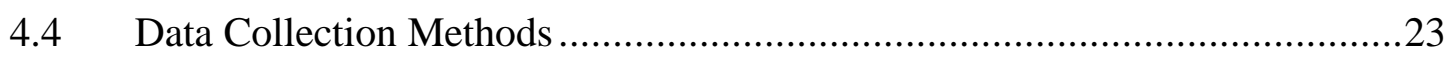

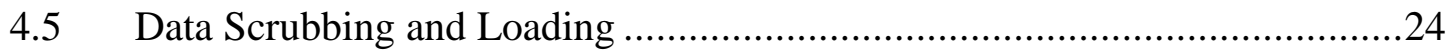

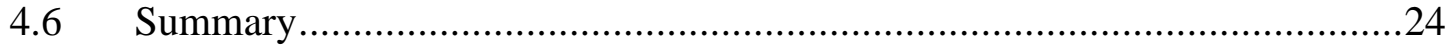

Chapter 5 - Implementation ..........................................................................................25

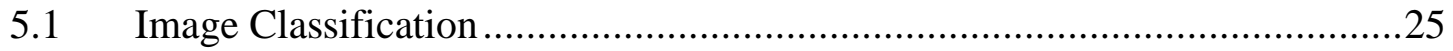

5.2 Analysis using Landscapemetrics .........................................................28

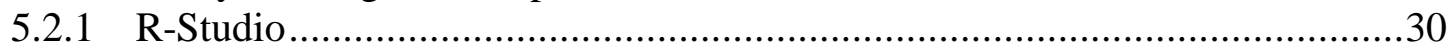

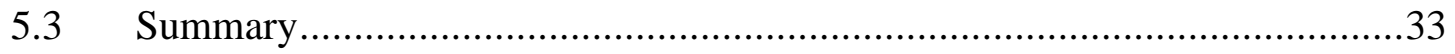

Chapter 6 - Results and Analysis ..................................................................................35

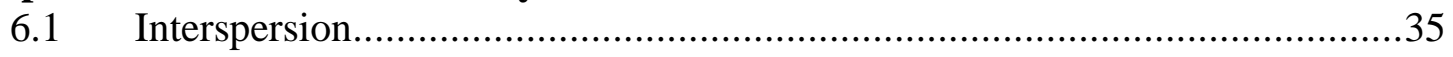

6.1.1 Landscape Level Interspersion .....................................................................

6.1.2 Class Level Interspersion..........................................................................

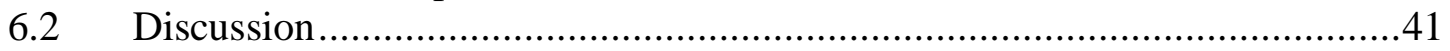

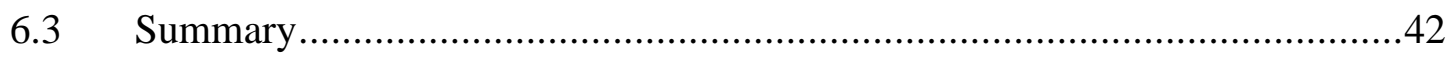

Chapter 7 - Conclusions and Future Work ....................................................................43

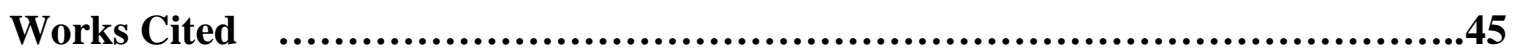


Appendix A. Raw Results .......................................................................................49

Appendix B. R-Script Using Landscapemetrics Library .................................53

Appendix C. Additional Class Level Charts......................................................56 


\section{Table of Figures}

Figure 1-1: Michigan Study Area.................................................................... 2 Figure 1-2: Wigwam Bay State Wildlife Area with Labeled Survey Points (NAIP

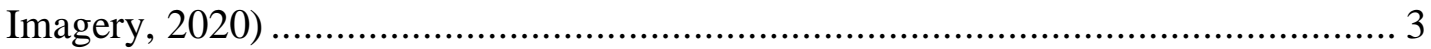

Figure 3-1: General Project Workflow .............................................................. 15

Figure 3-2: Source Imagery vs Provided NDVI Layer (June 2019) ........................ 16

Figure 3-3: 2018 Source Imagery used in this Project with Survey Points ............... 17

Figure 3-4: Task Break Down Flowchart-Parts 1 \&2 .......................................... 18

Figure 4-1: Project Conceptual Model ………………….................................... 21

Figure4-2: Logical Database Model ................................................................. 22

Figure 5-1: Image Selection for Classification …………................................. 25

Figure 5-2: 2018 Training Samples for Selected Images ...................................... 26

Figure 5-3: Wigwam Bay Wetland Study Area 2018 Classification Overview ....... 27

Figure 5-4: Focused Classification Around Survey Point WGIW-08 ...................... 28

Figure 5-5: Full Analysis Workflow Diagram ...................................................... 31

Figure 5-6: Part 1 of the Analysis Workflow....................................................... 32

Figure 5-7: Part 2 of the Analysis Workflow....................................................... 32

Figure 6-1: Landscape Level Interspersion Rate Change ...................................... 36

Figure 6-2: Study Area 6 Classification Change ................................................. 36

Figure 6-3: Study Area 6 Class Interspersion ........................................................ 37

Figure 6-4: Study Area 2 Class Interspersion .................................................... 38

Figure 6-5: Study Area 2 Classification Change.................................................... 39

Figure 6-6: Study Area 8 Class Interspersion ....................................................... 40

Figure 6-7: Study Area 8 Classification Change..................................................... 40

Figure 6-8: Survey Area 4 Class Interspersion ..................................................... 41

Figure 6-9: Study Area 4 Classification Change....................................................... 41

Figure C-1: Study Area 9 Class Interspersion ……………................................... 56

Figure C-2: Study Area 5 Class Interspersion ……………….......................... 57

Figure C-3: Study Area 7 Class Interspersion ……………................................ 57

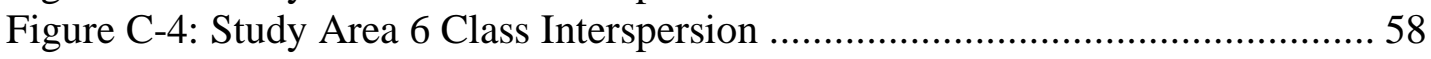

Figure C-5: Study Area 3 Class Interspersion …………...................................... 58

Figure C-6: Study Point 1 Class Interspersion..................................................... 59 


\section{List of Tables}

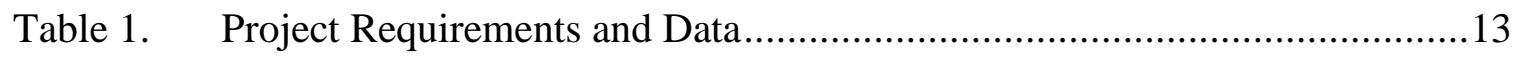

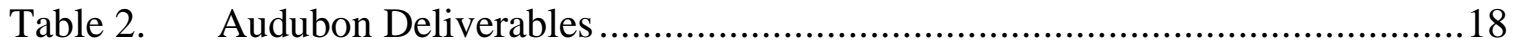

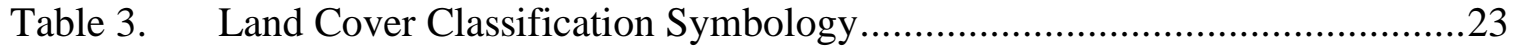

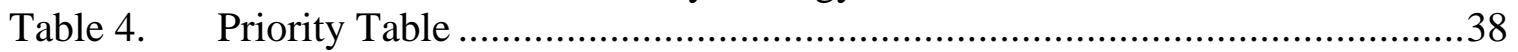

Table 5. Raw results from 2018..................................................................49

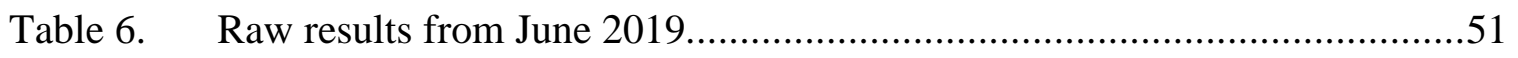




\section{List of Acronyms and Definitions}

$\begin{array}{ll}\text { Esri } & \text { Environmental Systems Research Institute } \\ \text { GIS } & \text { Geographic Information Science } \\ \text { GeoTiff } & \text { Geographic Tagged Image File Format } \\ \text { GPS } & \text { Global Positioning System } \\ \text { IJI } & \text { Interspersion and Juxtaposition Index } \\ \text { KMZ } & \text { Keyhole Markup Language Zipped } \\ \text { lsm } & \text { Landscape Metrics } \\ \text { NAD } & \text { North American Datum } \\ \text { NAIP } & \text { National Agricultural Imagery Program } \\ \text { NDVI } & \text { Normalized Difference Vegetation Index } \\ \text { RGB } & \text { Red, Green, Blue } \\ \text { TIFF } & \text { Tagged Image File Format } \\ \text { UAV } & \text { Unmanned Arial Vehicle } \\ \text { WGS } & \text { World Geodesic System }\end{array}$




\section{Chapter 1 - Introduction}

There is growing concern and evidence that the presence of invasive plant species negatively affects habitat quality for the native plants, especially when both organisms occupy the same or similar niches in an ecosystem. The displacement of native plants required for healthy bird habitats caused a cascade of negative reactions and declining bird populations (Gilsson, Brady, Paulios, Jacobi, \& Larkin, 2015).

Because of the negative relationship between birds and invasive plants, effort has been devoted to understanding how specific species respond to the changes in their environment by combining both direct and remote data collection and analysis techniques. In the past, researchers were hired in large numbers to go on foot into the field to record species presence and movement patterns. They carried a handheld GPS or Trimble, and a notebook or tablet. The collected data was then stored and organized, usually in a spreadsheet cleaned, and analyzed. The drawback was that it could take a very long time to collect an adequate amount of data for analysis. With the use of drone imagery and remotely sensed data, large areas can be investigated faster since drones can cover the same amount of area as a person but in a fraction of the time and cost. The digitized data can be combined with available direct observation data, yielding an analysis quickly and reliably.

The purpose of this project was to assess the spread of the invasive plant species phragmites Australis (common reed), cattail and other invasive plants. The client wanted a baseline habitat composition study and an assessment of the change, over two seasons at nine study areas in the Wigwam Bay State Wildlife Area, as shown using a green push pin in Figure 1-1 and a close up view in Figure 1-2. The client's overall objective was to identify the study areas with higher invasive species spreading rates, to target future conservation efforts. 


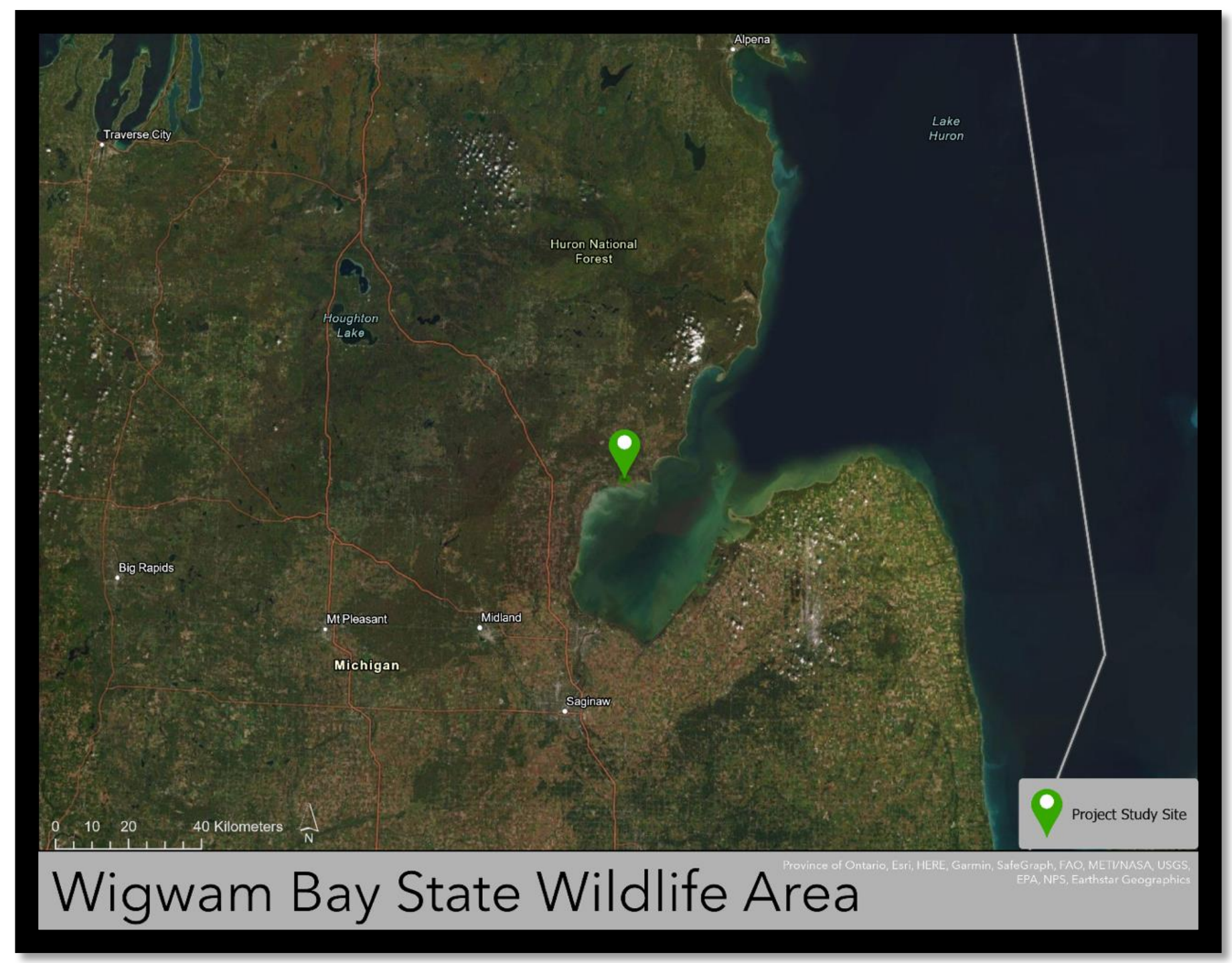

Figure 1-1: Michigan Study Area 


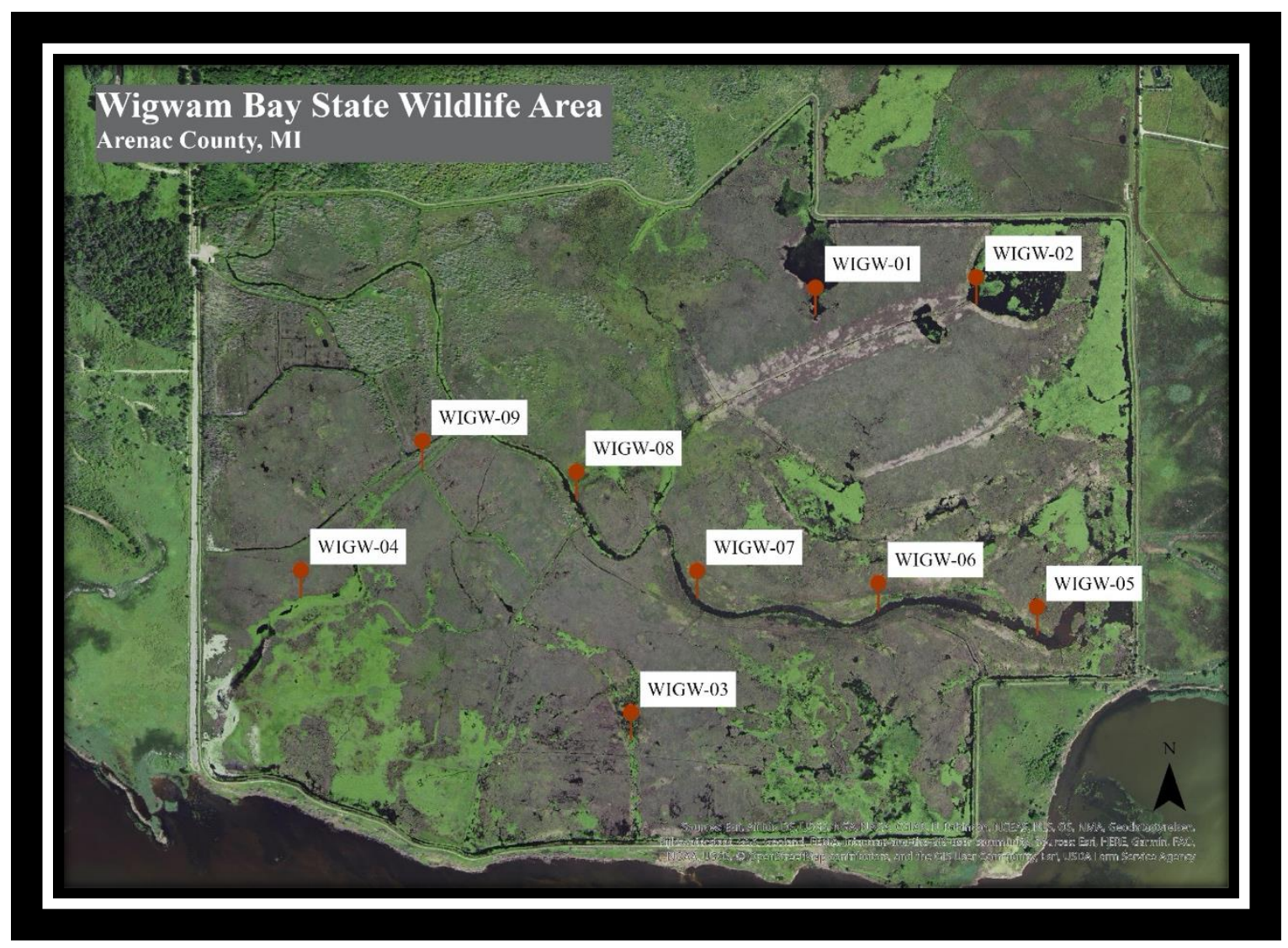

Figure 1-2: Wigwam Bay State Wildlife Area with Labeled Survey Points (NAIP Imagery, 2020)

\subsection{Client}

The client, the National Audubon Society, is a non-profit environmental organization focusing on conservation. The National Audubon Society is based in the United States and is one of the oldest non-profit conservation organizations in the country. The organization focuses primarily on birds and "uses science, education, and communitybased activities to promote its mission" (The National Audubon Society, 2020). Within the organization, there were two points of contact for the duration of the project, Stephanie Beilke, the Conservation Science Manager and Bradford Ksberg, the Wetland Restoration Manager. These individuals provided project data, defined objectives, and provided feedback throughout the project.

\subsection{Problem Statement}

Audubon was concerned about the spread of non-native plants in sensitive wetland habitats and how that might affect the dispersion of wetland birds. However, field work was too expensive and time consuming for a sustained and extensive study of the invasive plant spread. 


\subsection{Proposed Solution}

Imagery from Wigwam Bay State Wildlife Area was classified to calculate interspersion metrics, key measures to evaluate changes in the native birds' habitat due to non-native plants. The classification was completed following a supervised approach and the interspersion was calculated using the categorical output of the classification and survey points. Exact methodology and workflow are documented further in Chapter 3.

\subsubsection{Goals and Objectives}

The two main goals of this project were to create a model using GIS to classify raster images into water, mud, and multiple vegetation types and produce interspersion metrics. The technical deliverables were the classified images and interspersion calculations using R-Studio for each survey point across two summers, and the supporting documentation detailing the methods.

\subsubsection{Scope}

The deliverables requested by the National Audubon Society included ArcGIS files containing classified imagery and a final project report. The report contained an introduction, methods, results from the Interspersion and Juxtaposition Index (IJI), and a discussion section for processes used to complete the project including repeatable steps for future analysis by Audubon. The results of the imagery analysis included calculated interspersion metrics for nine marsh bird study areas (within $150 \mathrm{~m}$ of each of nine survey points) at Wigwam Bay State Wildlife Area in the Michigan Wetlands. The metrics were calculated at the landscape and class levels amongst four-Emergent Vegetation, Water, Exposed Mud and Floating Vegetation — out of an original six classes for 2018 and 2019.

\subsubsection{Methods}

Classification was conducted on imagery from the Wigwam Bay Wetlands collected in the years 2018 and 2019. The images were sorted and placed into mosaic datasets in ArcMap prior to the image classification. Next, several training datasets were created according to spectral similarity. The images were classified using the Maximum Likelihood classification method. This process was used for both the 2018 and 2019 datasets. The classification was comprised of six categories-Open Water, Exposed Mud, Floating Vegetation, Emergent Vegetation, Trees / Shrubs, and Other. Once the classification was finished, the layers were reclassified down to the four classes listed in Section 1.3.2 for analysis and exported as GeoTiff files.

Following the raster layer preparation, the survey points in the 2018 and 2019 June data were uploaded into R-Studio to create the interspersion rates using the landscapemetrics library suggested by the client. The calculation used the Interspersion and Juxtaposition Index (IJI) formula to evaluate the percentage of patch adjacencies between land category types. The exact methodology and parameters used in the project are discussed in more detail in Chapters 3 and 4. 


\subsection{Audience}

The intended audience of the report are professionals in GIS or environmental fields such as Ecology or Ornithology as this study deals heavily with birds and the assessment of the wetland habitat health. Professionals from the Audubon Society may also read this report.

\subsection{Overview of the Rest of this Report}

This report was organized into seven chapters including this introduction. Chapter 2 is the literature review, context, and background information about the impact of invasive plants on marsh bird habitats. The chapter was split into two major sections with the first focusing on the interspersion and the impact of invasive plants on marsh bird habitat and the second on the technical aspects, such as the analysis procedures used to conduct prior, related studies. Chapter 3 describes the system and overall project design and specifications, with database design specifications being discussed in Chapter 4 . The implementation plan is discussed at length in Chapter 5 along with explanations of adjustments made throughout the life of the project. The results and discussion are in Chapter 6. The last chapter, Chapter 7, details future work and research suggestions for future projects of a similar nature. The appendices contain additional relevant figures and tables including the full R-Script, analysis workflow and raw metrics output. 


\section{Chapter 2 - Background and Literature Review}

This chapter provides background explaining why evaluating habitat composition is important and results from prior studies. There are two subsections. The first subsection focuses on the effects of invasive species and habitat change on birds. The second subsection focuses on the technical aspects of this project - the use of remote sensing and classified imagery in conjunction with habitat analysis.

\subsection{Invasive Species and the Impact on Birds}

Gilsson et al., (2015) studied how three specific species of marsh birds were affected by invasive plants, the condition of the habitat, and whether it was intact or recently rehabilitated by people. The study showed that the bird species examined were heavily influenced by differences in plant community composition, habitat, and health of the vegetation (Gilsson, Brady, Paulios, Jacobi, \& Larkin, 2015). Two of the bird species were negatively influenced by the presence of invasive wetland grass reed canary grass, but a third species was positively influenced by the presence of cattail, another invasive species.

According to research by Gottschalk, Huettmann, and Elhlers (2005), there was evidence that the marsh bird species had higher occupancy in natural sites with diverse native-dominated plant communities. Because of the need for complex habitat structures, Liu et al., (2010) stated that there needs to be more land cover mapping and monitoring of sensitive bird nesting areas to ensure that the species do not become endangered due to the increased presence of invasive plants. It is because of the bird's dependence on stable and varied plants in their environment that Gilsson et al., (2015) recommended redoubling efforts to control reed canary grass and restore diverse, native-dominated plant communities in the Wisconsin region and elsewhere.

A related article by Tozer (2016) concluded a long-term study that collected data on 15 breeding marsh bird species and documented whether their numbers increased or decreased. Tozer found that 9 out of the 15 species decreased significantly whereas only one species went up in number. His results showed that the most negatively affected bird species would greatly benefit from conserving, restoring, or creating large wetlands. Lesser negatively affected birds would also benefit from those actions or, as Gilsson et al., (2015) suggested, creating robust, emergent dominated, and native rich wetlands.

Tozer (2016) mentioned that specific invasive plant species of concern should be removed from the landscape if possible. Tozer studied purple loosestrife and phragmites Australis (common reed). Phragmites is an extremely invasive plant that was mentioned in many articles addressing wetlands or freshwater environments including Ketterning, Mock and McKee (2016). It is a bamboo like plant that can grow to be six to nine feet tall with a reddish-brown plume on top when mature. It looks very similar to another reed species - arundo Donax (Giant reed), but Phragmites is a much smaller plant, and its leaves grow in a more spiral pattern around the stocks, unlike Arundo whose leaves grow more like a cornstalk.

The process and specifics of the expansion of Phragmites into wetlands was discussed by Chambers, Meyrson and Saltonstall (1999). Their study emphasized the 
disturbance of hydrologic cycle and nutrient regimes. A major concern further expressed by the authors was that the Phragmites expansion into tidal freshwater wetlands resulted in a reduction in biodiversity since many native species of plants were replaced. Along with the shift in the available native plant habitat was a reduction in insect, birds, and other types of animals.

The effects of Phragmites on marsh birds was researched in detail by Gagnon et al., (2014). The specific genotype of common reed currently invading the freshwater marsh was from Eurasia (Gagnon Lupien, Gauthier, \& Lavoie, 2014). Their research conducted a fixed-distance point count for some songbirds and visual surveys for water-birds native to their southern Quebec study site. The focus of their study was to evaluate the effects of the plant on native species in the area. They found that the impact of Phragmites varied depending on the species of bird and their needs. Some, like the songbirds, were generally adaptable and were not as affected. Wetland specialists - such as the Marsh wren, were affected in terms of nesting habitat. In their results, water depth was found to have more effect on marsh bird species and abundance of individuals and nest locations than the plant community.

There were structural differences between Phragmites stands relative to marsh vegetation plant communities dominated by other plant species such as bulrushes and sedges. According to Scarbrough, et al., (2013), Phragmites stands can still function as feeding and reproduction sites. However, it is also possible that in southern Quebec, the number and extent of common reed population had not yet reached the threshold beyond which negative effects could be significant.

\subsection{Species Interspersion and Calculation}

The Audubon society was interested in examining interspersion at its study site. While interspersion is a key habitat measure related to marsh bird abundance, it is not easily quantified (Rehm \& Baldassarre, 2007). Rehm and Bladasserre used the FRAGSTATS software program and the IJI along with aerial photos to measure the interspersion within wetlands as meters per hectare of interface between water and vegetation - a measure of how blended the landscape is. Highly interspersed marsh wetlands increase breeding habitat diversity and density of bird species, making interspersion a critical metric for wetlands habitat.

There was an alternative to the formula used by Rehm. Known as the Contagion Index, it was proposed in the 1980's (McGarigal \& Marks, 1995). The contagion formula was more sensitive to both dispersion and interspersion, and evaluated interspersion based on the cell value rather than patch type as in IJI (McGarigal \& Marks, 1995). The IJI, in contrast to contagion, is affected only by patch type interspersion and not necessarily by the cell size, contiguity, or dispersion of patches. Because of this, though often indirectly affected by dispersion, the IJI directly measures patch type interspersion (McGarigal \& Marks, 1995). Another difference with the contagion index is that a smaller grain size will result in greater contagion because of the proportional increase in like adjacencies from internal cells - which are ignored when using the IJI formula. The IJI is not affected this way because it only considers patch edges not individual cell assignments (McGarigal \& Marks, 1995).

The IJI formula is compatible with raster layers and can be computed at both class and landscape levels. The formula evaluates patch adjacency with all other patch types. 
Like adjacencies are not evaluated with this index because a patch cannot be adjacent to the same type of patch - two or more of the same classes are counted as one. The landscape level IJI defined in the R-Studio library landscapemetrics is presented in Equation-1 below and was originally defined by McGarigal and Marks (1995).

$$
I J I=\frac{-\sum_{i=1}^{m} \sum_{k=i+1}^{m}\left[\left(\frac{e_{i k}}{E}\right) \ln \left(\frac{e_{i k}}{E}\right)\right]}{\ln (0.5[m(m-1)])} * 100
$$

In the equation the $e_{i k}$ are the unique adjacencies of all classes (lower/ upper triangle of the adjacency table - without the diagonal). The $E$ refers to the total length of the edges within the landscape or study area patches in question (Hesselbarth, Sciaini, With, Wiegand, \& Nowosad, 2019). The $m$ is the number of classes or land cover types. IJI is an aggregation metric — or "salt and pepper" metric and is reported as a percentage. The result approaches $0 \%$ if a class is only adjacent to a single other class - such as if there is only a solid border between a dirt road and a corn field (McGarigal \& Marks, 1995). Conversely when the result approaches $100 \%$ interspersion then every class within the study area is equally adjacent or in contact with all other classes being considered or contained in a classified image.

In addition to the landscape level interspersion there is another juxtaposition formula for class level analysis. The class level equation is very similar to the landscape level, with the same parameters and type of result. The equation from Sciaini et al., (2019) is presented in Equation-2; this equation was also originally defined by McGarigal and Marks (1999).

$$
I J I=\frac{-\sum_{k=1}^{m}\left[\left(\frac{e_{i k}}{\sum_{k=1}^{m} e_{i k}}\right) \ln \left(\frac{e_{i k}}{\sum_{k=1}^{m} e_{i k}}\right)\right]}{\ln (m-1)} * 100
$$

As with the landscape level version of the equation, the $e_{i k}$ refer to the unique adjacencies of all classes (lower/upper triangle of the adjacency table - without the diagonal) and $m$ is the total number of classes (Sciaini, Hasselbarth, With, Wiegand, \& Nowosad, 2019).

Including the class level metric allowed for a deeper analysis on observed annual changes. As with the landscape level metric, the class level IJI function measures the interspersion of different patches in the landscape. Low values from 0-50\% represent clumped or an overall disproportionate distribution, whereas higher values $50-100 \%$ mean that the patches are increasingly uniformly mixed or adjacent to each other.

\subsection{Land Cover and Species Mapping with Remote Sensing}

Hanson (1975) explained the importance of plant communities as part of good habitat management. Although the original paper focused on range land plants like prairie grass, the main principles Hanson published are applicable in most other contexts in land cover 
mapping. Among many elements discussed for landcover classification, Hanson (1975) used five common criteria to classify vegetation in his study: physiognomy, habitat relations, geographic distribution, successional statue, and floristics.

Xie et. al. (2008) compared popular remote sensing devices, commonly adopted image processing methods and classification accuracy assessments. Their comparisons for vegetation mapping discussed various processes, considerations, and techniques. Imagery of different sources have differences in spectral, spatial, radioactive, and temporal characteristics, and are desirable for different types of vegetation mapping. This was discussed at length in the review and examples were given regarding when to use traditional approaches vs. remote sensing for land cover mapping.

Similar wetland mapping using imagery was addressed in a later study by Xie, Zhang, and Welsh (2015), with an emphasis on Phragmites using public remotely sensed images. Using the publicly available, remotely sensed images to map wetlands and invasive species was attractive due to its low cost. However, the analysis was challenging because of the variability of mapping accuracy. In their study, the researchers came up with an innovative wetland and invasive plant mapping technique that made use of three integrations: feature extraction, integration of high spatial-resolution images with high spectral resolution, and the use of field data with the integrated and classified images (Xie, Zhang, \& Welsh, 2015). This technique helped to integrate the available imagery and produced an accurate invasive species map of the study area.

The research conducted by Pickens and King (2014) investigated the ability of non-static and multi-temporal habitat characteristics to predict marsh bird distribution, while also testing the model generality and transferability between coastal habitats. The authors used Landsat images to quantify annual conditions and cumulative, multitemporal habitat characteristics. At the end of the study, Pickens and King's (2014) models found that dynamic ecological processes could affect species distribution and that habitat relationships differed within dominant landscape characteristics. There was some discrepancy in the quality of the imagery used since not all of it was at an appropriate scale to give the detailed result Pickens and King sought.

A general overview of remote sensing applications in classifying and mapping wetland vegetation was compiled and analyzed by Elhadi, Mutanga, and Rugege (2010). The authors argued that remote sensing had major challenges requiring consideration to achieve successful results. Some of the considerations in the review were: selecting the appropriate spatial, spectral resolution, and processing technique to use to extract information on the wetland vegetation and water nearby. Elhadi, Mutanga and Rugege (2010) explained that wetland plants were not as easy to detect compared to typical terrestrial plants which tend to be more stratified. This challenge was due to a few reasons, one being that wetland vegetation contains high spectral and spatial variability because of steep environmental gradients which produce short ecotones and indistinct edges between types of vegetation. The second issue with wetland vegetation was that the reflectance spectra of the canopies can be very similar and are combined with soil, hydrologic regime, and atmospheric vapor (Elhadi, Mutanga, \& Rugege, 2010). But as stated in the authors' introduction, hyperspectral narrow channels may have the ability to detect and map the spatial heterogeneity of wetland vegetation-helping to eliminate some of the difficulties presented when dealing with the vegetation. 
Texture is another type of information sometimes used in classification in place of the traditional pixel-based techniques, although it requires more complex data layers to execute accurately (Haralick, Shanmugam, \& Distein, 1973). The texture approach was used in several studies including the ones by Haralick, Shanmugam and Distein (1973) and by Bellis, et al., (2008). Texture is necessary sometimes because the more commonly used land cover classifications do not always capture the variability within a given class which may hold important information about the habitat in question (Bellis, et al., 2008). Texture measures, derived from remote sensing images, can quantify the variability in habitat features and types (Bellis, et al., 2008). Because of this ability, texture-based classification is becoming a more readily used medium to classify complex land areas and generate habitat models.

Classification and land cover mapping techniques start with the imagery sourcei.e. satellite or UAV (unmanned aerial vehicle), or airplane via classic photogrammetry. The source often dictates the price and the quality of the imagery in question. Satellite imagery will often be readily available and sometimes free, may have many bands to choose from and has high spectral resolution, but the pixel sizes tend to be large (Klemas, 2011). This may be sufficient in some cases, but as stated previously, wetland mapping tends to happen at a smaller scale and requires a much smaller pixel size to account for individual species as needed. Therefore, land cover mapping tended to be done with UAV's which more often meet the criteria (Samiappan, et al., 2017). Using a drone also provides more control over when the data could be collected, as compared to scheduled satellite passing. Timing matters for the elimination of issues such as clouds and shadows. Cost effective UAV imagery does not have as may bands as a satellite, but if infrared bands are included, other features common with satellite data such as panchromatic or ultraviolet are not needed. If the study area in question is large, then the lower resolution imagery from satellites may be more useful to avoid long run times and the relatively more expensive collection and processing via UAV imagery.

In the case of wetland classification and analysis - especially in the case of very local applications, UAV imagery has been used far more frequently. Maraccio, Markle and Chow-Fraser (2016) compared the ability of two common versions of UAVs-fixed wing and multi-rotor, to determine if there was a difference in quality and ability to map dynamic changes in a marsh. The data collected via the two UAVs were compared to available "digital aerial true color, high resolution photographs provided by the government that were taken every five years in the spring" (Marcaccio, Markle, \& ChowFraser, 2016). They found that fixed wing system produced the most effective data for determining percent cover of floating and emergent vegetation $(58 \%$ and $64 \%$ overall accuracy, respectively). Both the fixed wing and the multi-rotor drones were comparable in their ability to determine dominant habitat types (moderate kappa agreement) and were superior to the available satellite imagery in this respect (poor kappa agreement). The authors' conclusion from their study was that UAVs can provide a time-sensitive, flexible, and cost-effective option to capture dynamic seasonal changes in wetlands (Marcaccio, Markle, \& Chow-Fraser, 2016).

A review of successful remote sensing applications in wetland vegetation mappings by Mutanga and Rugege (2010) provided fundamental information regarding the spectral characteristics of wetland vegetation and other major components related to wetland studies like biomass and leaf area index, making it evident that remote sensing 
projects focusing on wetlands have unique and complex challenges to overcome and need careful consideration to obtain successful results. (Mutangs \& Rugege, 2010). The factors detailed by the authors included an in-depth understanding of the interaction between electromagnetic radiation and wetland vegetation in an environment, selecting the appropriate spatial and spectral resolution, as well as choosing a suitable processing technique.

\subsection{Summary}

There is a negative relationship between the spread of invasive plant species such as the Common Reed and Cattails and the health of wetland habitats like those present in the current study area (Wigwam Bay State Wildlife Area). Many of the approaches in these past studies included the use of either semi-public satellite imagery such as those from LANDSAT or Quickbird. Others used drone imagery taken specifically for their own uses, or a mixture of both. Nearly all used the infrared band and the near infrared bands for much of their classifications.

For wetlands, there have been many similar projects and studies that mapped the presence of the invasive species and discussed the classification methods that can be useful when processing imagery for wetlands including infrared. More recently, some analysts have used texture-based classification to identify the individual plants in imagery. These studies proved that while texture classification is very useful for classifying plant species, texture classification is only possible in landscapes where textural differences are very distinct. 


\section{Chapter 3 - Systems Analysis and Design}

This chapter describes project analysis and design in detail. This chapter also includes the imagery challenges encountered during the project that affected the final design.

\subsection{Problem Statement}

Audubon was concerned about the spread of non-native plants in sensitive wetland habitats and how that might affect the dispersion of wetland birds. However, field work was too expensive for a sustained and extensive study of the non-native plant spread.

\subsection{Requirements Analysis}

The client deliverables for this project were GIS files containing classified imagery and a report containing introduction, methods, results, and discussion sections. The project required imagery analysis using interspersion calculations within 150 meters of each bird survey point for a total of nine study areas. To conduct this analysis, the client provided imagery from 2018 and 2019 and the survey points. Functional and non-functional requirements can be found in Table 1 .

\section{Table 1. Project Requirements and Data}

\begin{tabular}{|l|l|l|}
\hline Deliverable & Functional Requirements & $\begin{array}{l}\text { Non-Functional } \\
\text { Requirements }\end{array}$ \\
& $\begin{array}{l}\text { Two continuous layers for } \\
\text { each year with source } \\
\text { imagery } \\
\text { Must contain six classes: } \\
\text { Open Water, } \\
\text { Exposed Mud, }\end{array}$ & $\begin{array}{l}\text { ArcMap 10.7 or later } \\
\text { Floating Vegetation, } \\
\text { Emergent Vegetation, }\end{array}$ \\
& $\begin{array}{l}\text { Image Analyst } \\
\text { Trees/ Shrubs, } \\
\text { Other }\end{array}$ & Spatial Analyst \\
& & \\
\hline
\end{tabular}




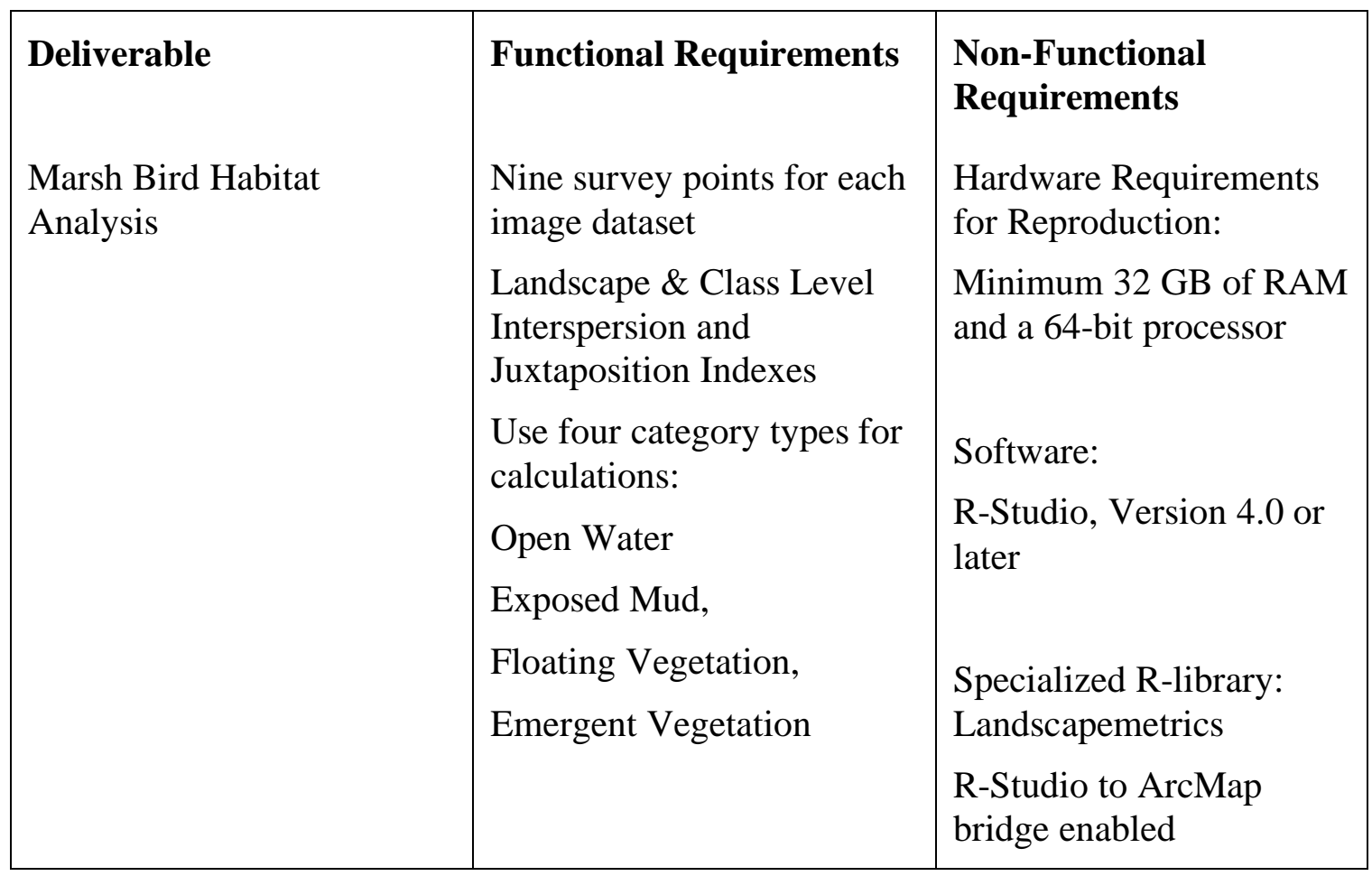

To produce the functional requirements - classified images and interspersion results, the software detailed above was required. The client specified that the classification task be submitted in ArcMap 10.7 or later format. To use R-Studio, the open source analysis software preferred by the client, the following libraries were downloaded and enabled: arcgisbinding, landscapemetrics, raster, gdalUtils, sp and dplyr. The landscapemetrics library was used to calculate interspersion metrics. The other libraries were necessary to manage the raster data and point features in R-Studio.

\subsection{System Design and Imagery Related Decisions}

Drone imagery collected and provided by the client was used to analyze the composition of the vegetation within the Wigwam Bay Wetland area. The imagery, originally collected in the summers of 2018 and 2019, was mosaiced together and classified into six categories for the first deliverable and then reclassified down to the four chosen categories prior to analysis. The survey points provided by the client defined the study areas and were inputs to the interspersion calculations. The data and project overview 
workflow are shown in Figure 3-1.

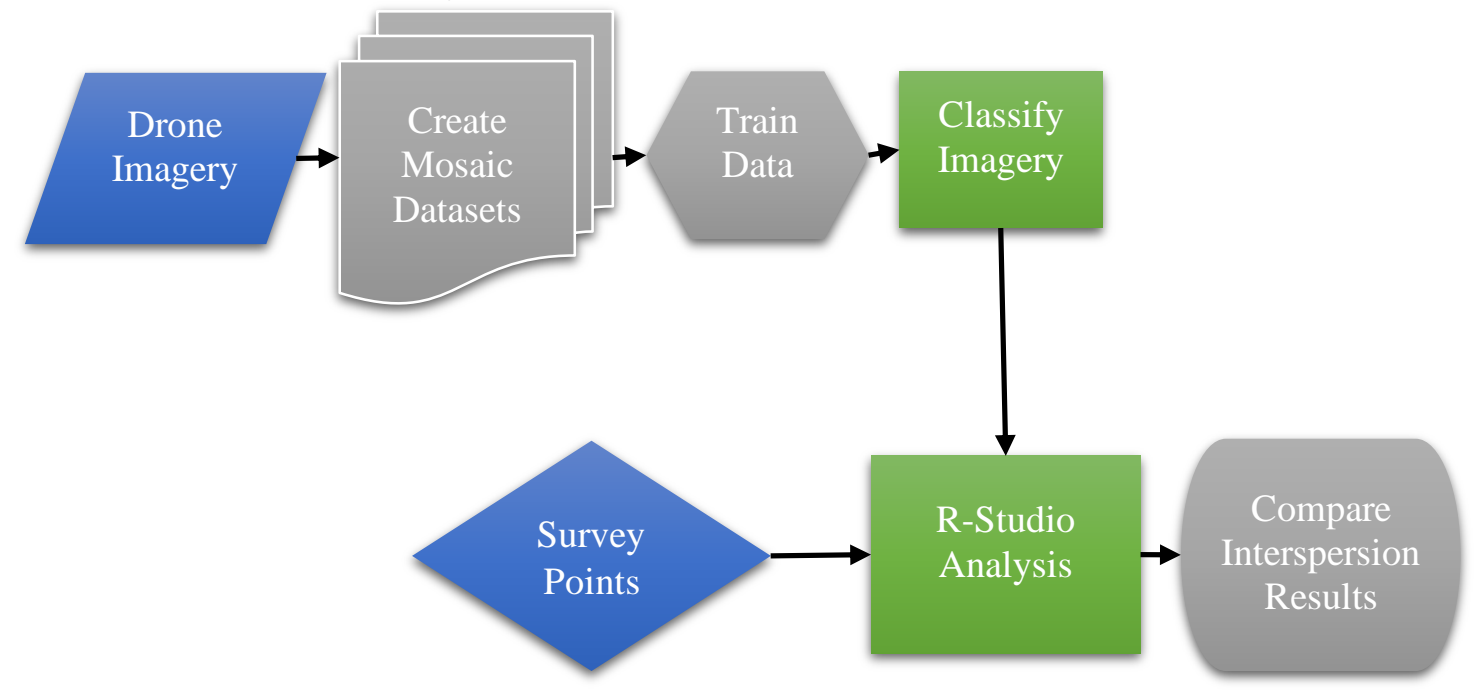

\section{Figure 3-1: General Project Workflow}

The drone imagery received was comprised of about nine images per dataset with one dataset for 2018 and two datasets for 2019, one for June 2019 and one for July 2019 for a total of 24 images. The imagery consisted of individually flown, developed, and processed orthophoto imagery captured at different times of day. While processing the imagery it became apparent that twelve of the images did not align correctly. The Audubon was informed about this issue early in the project and decided that this did not invalidate the analysis because the interspersion analysis buffers (study areas) were mainly in the center of each image, away from the image boundaries, so the misalignment was not relevant for the interspersion analysis.

The NDVI calculated imagery presented multiple challenges and the lack of infrared band presented multiple challenges. The 2019 imagery included NDVI calculated imagery, but it was a different pixel size from the orthophoto images. For both years, the image extents were different. Also, the NDVI layer colors from the 2019 dataset were not very well defined: the same colors appeared for both vegetation and water areas, as shown in Figure 3-2. This was an issue for the project because similar colors mean they have similar NDVI values and distinctive values were necessary for classification.

Lastly, none of the orthophoto images included an infrared band, making species specific vegetation distinction unreliable. The challenges presented in the provided data layers led to the decision to use the emergent vegetation as an invasive species proxy and to use the provided orthophoto imagery from the 2018 and 2019 imagery for classification and analysis. 


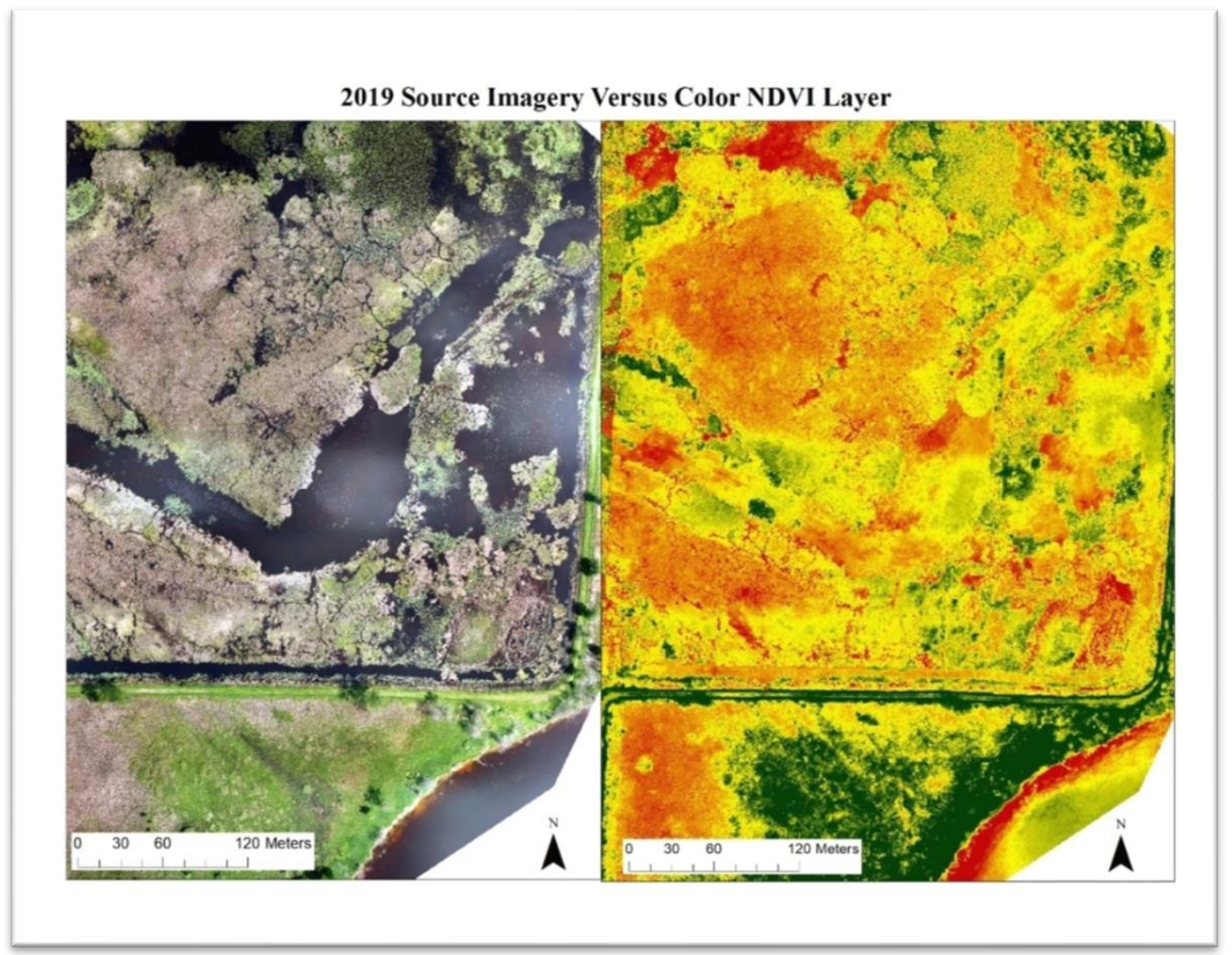

Figure 3-2: Source Imagery vs Provided NDVI Layer (June 2019)

The orthophoto imagery also presented challenges due to their spectral characteristics. The range of colors within each image is considerably different because the drone flights were at different times of day. Additionally, water reflections create glare, which is common with wetlands data gathered in bright sun conditions. The spectral characteristic differences are visible in the source imagery from 2018 shown in 
Figure 3-3.

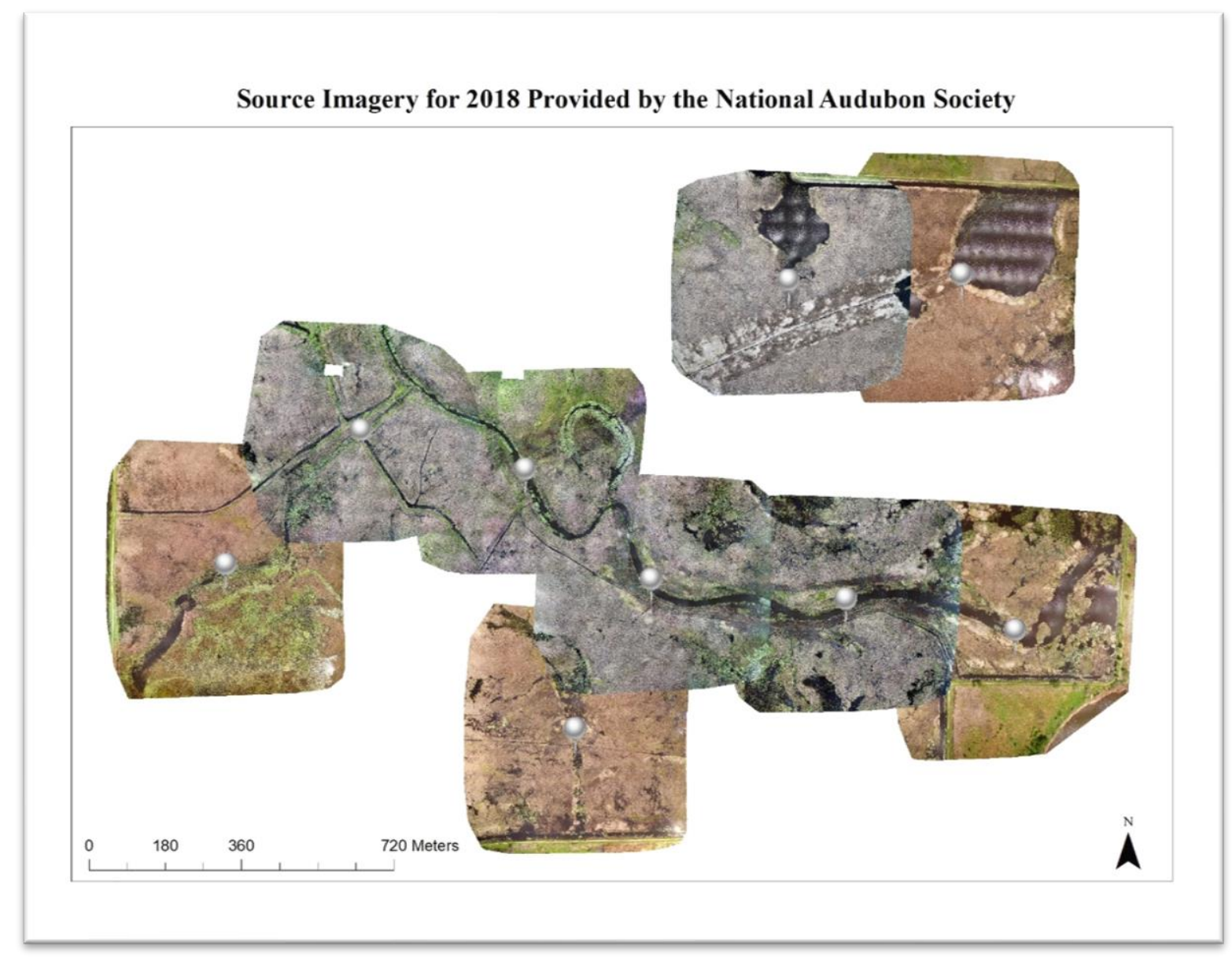

Figure 3-3: 2018 Source Imagery used in this Project with Survey Points

To address the spectral challenges the datasets were divided into different groups according to their spectral characteristics and the classification was conducted on each group separately.

The final challenge was related to ground truth collection. Audubon provided data collected for a prior vegetation composition study. The points provided had multiple vegetation types assigned, essentially creating a one to many relationship instead of the one point to one class relationship required for accuracy assessments. To address this, a visual check between source imagery and classification layers was used, with the client's approval. Collectively, these challenges led to the final project plan. The methods used to train and classify the compiled image datasets are explained in the next section.

\subsection{Project Plan}

There were three major tasks to complete this project, identified below as Tasks 1,2 and 3 . The three tasks correlate with the deliverables requested by Audubon, also shown below in Table 2. The steps for the two technical tasks 1 and 2 are shown in Figure 3-4 and followed by additional detail. 
Table 2. Audubon Deliverables

\begin{tabular}{|c|c|c|c|}
\hline Task & Deliverable & Type & Format \\
\hline 1 & Image Classification & Data & File Geodatabase \\
\hline 2 & R-Script & $\begin{array}{l}\text { Support } \\
\text { Documentation }\end{array}$ & $\mathrm{R}$ file \\
\hline 3 & $\begin{array}{l}\text { Marsh Bird Habitat } \\
\text { Analysis Report }\end{array}$ & Documentation & $\begin{array}{l}\text { Word or PDF } \\
\text { document with } \\
\text { introduction, results, } \\
\text { discussion sections }\end{array}$ \\
\hline
\end{tabular}

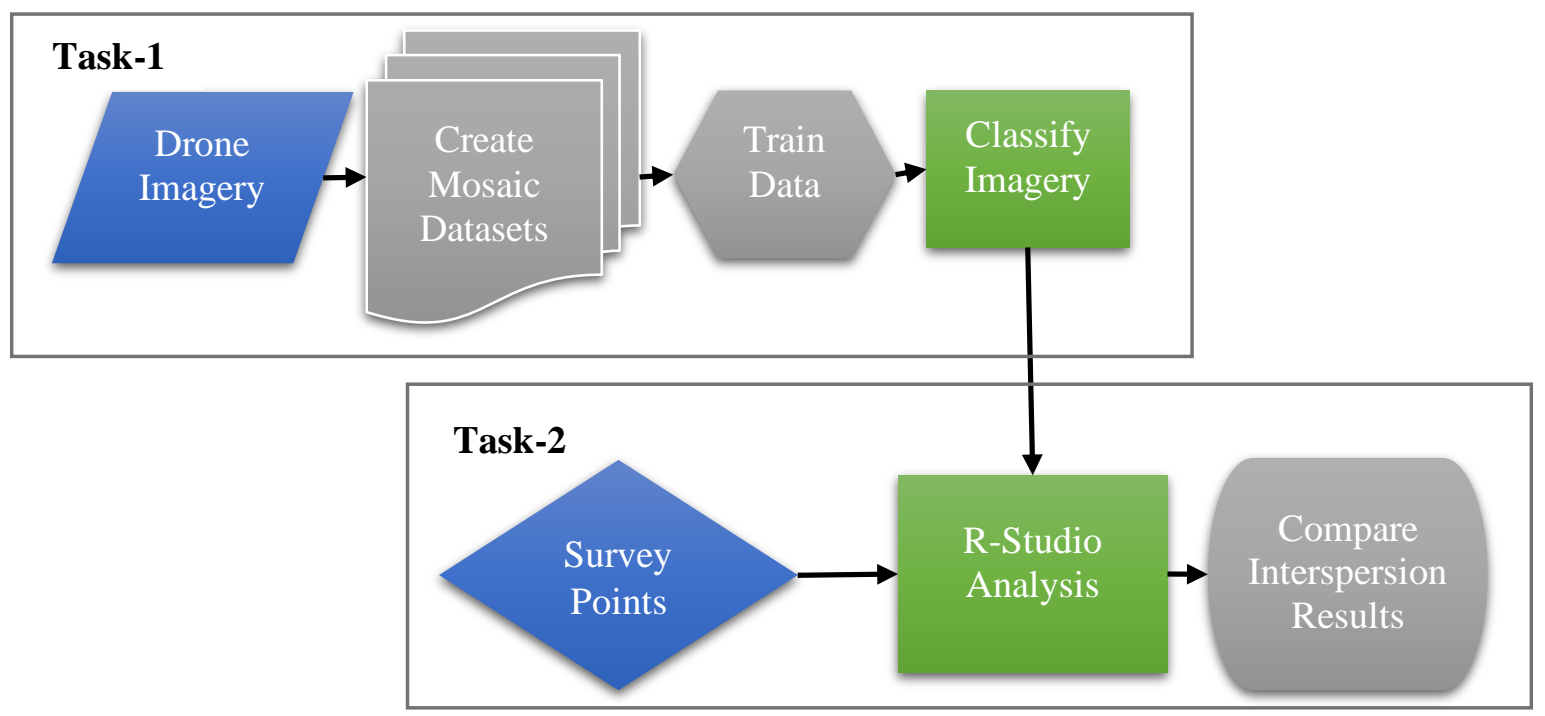

Figure 3-4: Task Break Down Flowchart-Parts 1 \&2

Task 1 - Classify Imagery

For this task, the provided imagery from the client was placed into mosaic datasets by year. Next, two unique training datasets were developed to avoid spectral confusion and error as much as possible. The classification categories are Open Water, Emergent Vegetation, Floating Vegetation, Exposed Mud, Trees/Shrubs and Other as agreed to with the client. During the process of creating training samples, the interactive classification tool from the tool bar was used to expedite the process. This tool uses the Maximum Likelihood Methodology.

After classification, the images were reassembled with the mosaic tool, reclassified to avoid duplicate feature classes, and layered on top of the source imagery. The quality check for this task was a visual check against the original source imagery followed by acceptance from the client. Once the classification layers were complete, each layer was reclassified to only four classes including: Open Water, Exposed Mud, Floating Vegetation and Emergent Vegetation. The reclassified layers were then exported as GeoTiff layers for Task 2.

Task 2 - Analyze Imagery and Compute Habitat Model 
The analysis task brought the final classified imagery in GeoTiff format into $\mathrm{R}$ to compute the interspersion rates using the "landscapemetrics" library. Specifically, the 'sample_lsm' tool was used. In the original client's proposal, the interspersion calculation was to consider only two classes: Open Water and Emergent Vegetation, but the IJI formula requires at least three for the function to work. Therefore, Exposed Mud and Floating Vegetation were added. Both are major components of the wetland habitat, readily visible on the imagery, and both impact habitat health. Interspersion metrics results were written to Microsoft Excel files, where the study area results were compared for changes from 2018 to 2019 at the landscape and class levels.

Task 3 - Documentation

The client requested a written report including a discussion section for the classification and analysis procedures as well as the scripts and tools used to get the results. This information is the same as presented in this thesis. The R-Script was thoroughly commented for clarity so that Audubon staff can reuse the code.

\subsection{Summary}

This project consisted of three tasks, each with an associated deliverable: classified imagery, an R-Script, and a written report with habitat metrics. The imagery used in this project was collected and processed by the Audubon and their partners. The orthophoto layers were used to create the classification layers with the six classes: Open Water, Floating Vegetation, Emergent Vegetation, Tree / Shrub, and Mud and Other. The classification layers were then reclassified down to four classes for analysis. Patches in each study area were then analyzed using the IJI definition in a R-Library tool and evaluated for compositional change over the two years. Results were delivered to the client along with the rest of the deliverables previously mentioned. The components of the geodatabase, symbology, data management techniques utilized, and organizational decisions made during this project are expanded upon in the following chapter. 


\section{Chapter 4 - Database Design}

This chapter reviews the conceptual and logical data models for this project in sections 4.1 and 4.2. All the data used in this project was preprocessed to finalized imagery by the client.

\subsection{Conceptual Data Model}

The conceptual model explains the entities and their relationships to this project. Changes in marsh habitat composition can indicate the spread of invasive species and the potential negative impact on marsh bird nesting habits is the motivation for this project. Audubon and their partners need to know where the invasive plants are spreading to prioritize conservation efforts in the area. Figure 4-1 illustrates the conceptual model of Interspersion and its relation to other habitat components within the project.

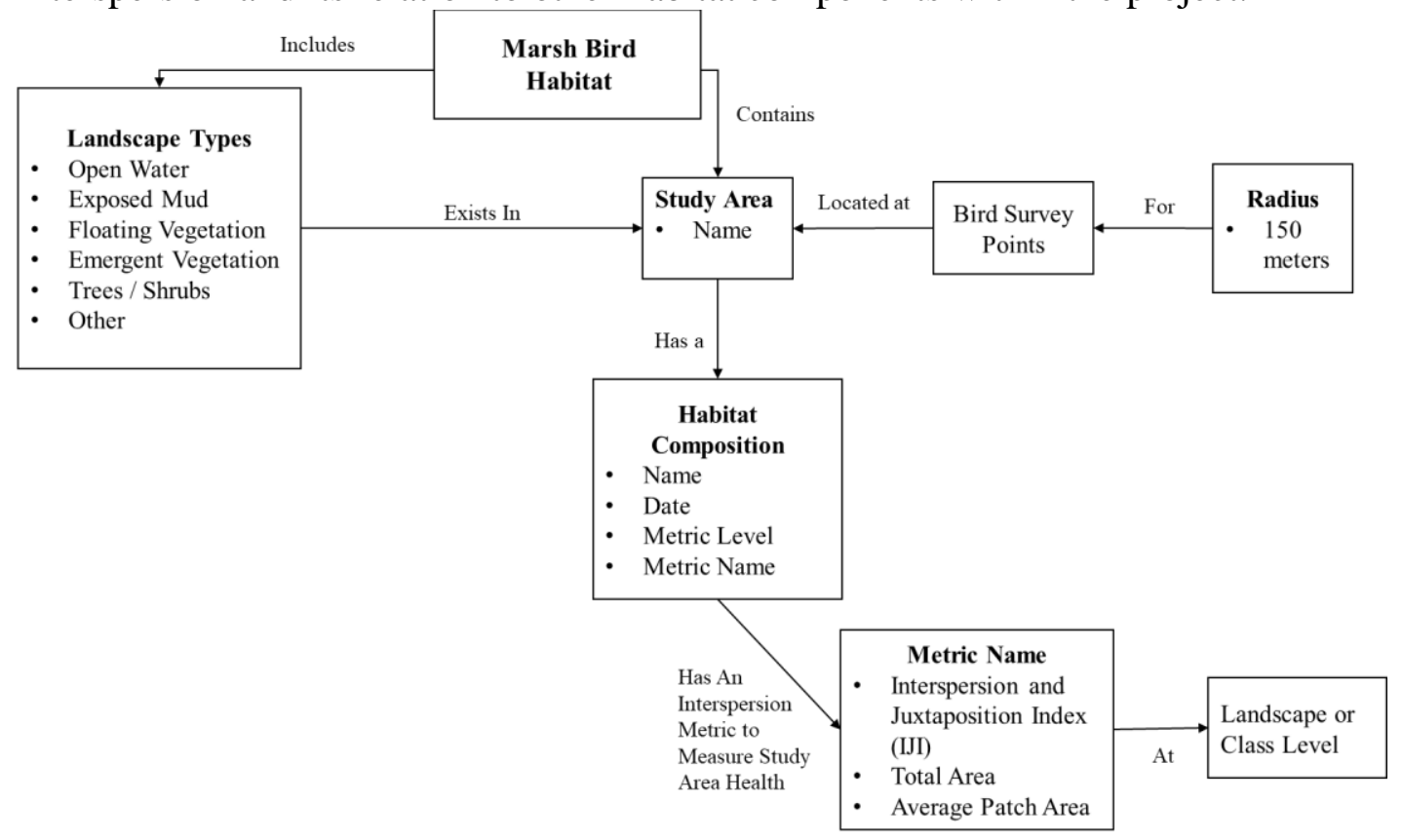

Figure 4-1: Project Conceptual Model

Wetland birds need a complex habitat with all the land types used in the classification layers apart from the Other category, which included man-made infrastructure such as roads. The study areas contained in the wetland and defined by a $150 \mathrm{~m}$ radius centered at the bird survey points and had a one to many relationship with the landscape types within their respective areas. The habitat health of the study areas is based on the landscape types present and is evaluated by the interspersion metrics. Areas with lower interspersion were a greater concern for further investigation by Audubon personnel. Where interspersion is high or increased, concern and priority level was lower. 


\subsection{Logical Data Model}

Drone imagery was collected by the client to analyze the composition and structure of vegetation within the Wigwam Bay Wetland area. The imagery originally collected in the summers of 2018 and 2019 were mosaiced together and classified using six categories prior to analysis. The classified data was needed to evaluate the interspersion in the nine study areas. The client provided two datasets for 2019, one completed in June and the other in July. Only the June dataset was needed for the analysis. There were about nine individual images per dataset. The logical database model for this project is shown in Figure 4-2.

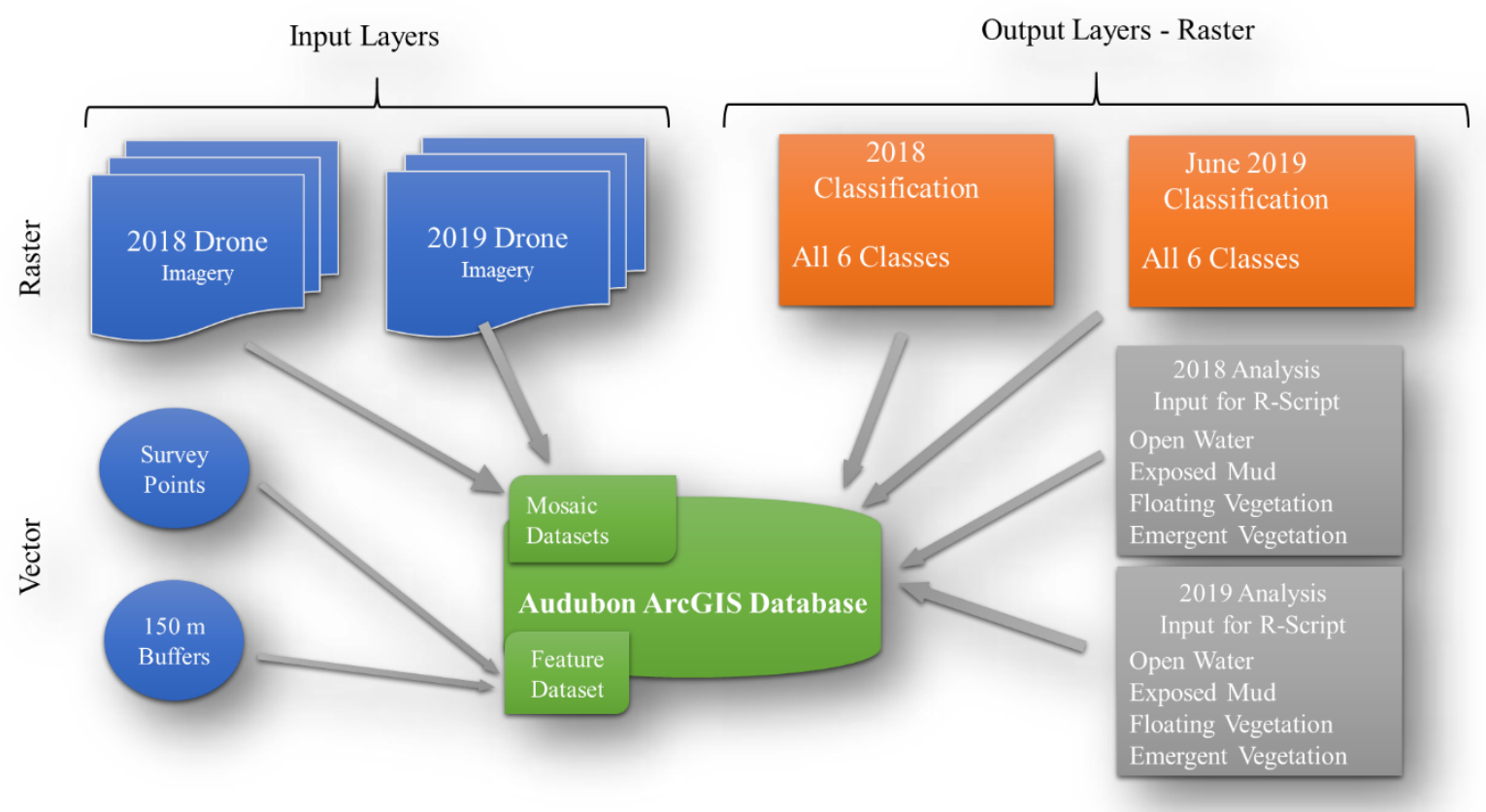

Figure4-2: Logical Database Model

The logical data model was straightforward with few complications as much was defined by the deliverables required. The blue input layers in Figure 4-2 were provided by the client. Orange represents Task 1 of the project deliverables. Output raster layers in grey are intermediate output layers. These intermediate layers are used later in the analysis procedure outlined in Chapter 5. All the analysis layers were derived from the two classification deliverables in orange in Figure 4-2.

All the components were stored in a 10.7 version file geodatabase with the survey points and the buffer layer being stored in a feature data set. Storing the vector layers in a feature dataset allows for more functionality than if the layers were stored separately.

The categorical layers include the classification of images stored in mosaic datasets according to the year of the source data collection. All the categories have the same symbology to make comparison simpler shown in Table 3 . The resolution of the original orthophoto images was very high - about $0.27 \times 0.27$ meters, as they were gathered using drones. The classifications are accurate at a small scale although spectral differences remain an accuracy concern. 
Table 3. Land Cover Classification Symbology

\begin{tabular}{|c|c|c|c|c|}
\hline Land Cover Type & Color & Red & Green & Blue \\
\hline Open Water & & 0 & 77 & 168 \\
\hline Exposed Mud & & 115 & 76 & 0 \\
\hline Floating Vegetation & & 255 & 255 & 0 \\
\hline Emergent Vegetation & & 112 & 168 & 0 \\
\hline Trees/Shrubs & & 0 & 115 & 76 \\
\hline Other & & 130 & 130 & 130 \\
\hline
\end{tabular}

There were elements in the model that were not used in the final analysis and comparison - like the buffers - but these were useful in the planning and visual checking necessary to ensure the that classified imagery and underlying data were processed correctly.

\subsection{Data Sources}

The raster imagery was processed internally by the client into individual orthophotos for each flight. The images were shared through a cloud database as TIFFs. All the 2018 and 2019 imagery layers use the geographic coordinate reference GCS WGS 1984 and the projection WGS 1984 UTM Zone 17N.

The classification used the 2018 and June 2019 orthophoto data as described in Project Plan 3.4.

The survey points used to make the analysis buffers were created using the protocol "Standardized North American Marsh Bird Monitoring Protocol (Conway, 2011)." This was developed by the U.S Fish and Wildlife as a standardized protocol for measuring marsh bird breeding and nesting patterns. The client uses this standard for all bird surveys and to verify data accuracy and validity.

\subsection{Data Collection Methods}

Data collection was very limited for this project as the client provided almost everything required. The only layer acquired from a source other than the client was an imagery basemap layer to use as a reference when making the classification training set near the edges of the provided drone imagery. This was NAIP imagery from the Esri Living Atlas. It was brought into ArcMap and clipped down to encompass just the outer-ring road around the Wigwam Bay refuge area (Figure 1-1). This layer was also used to make a study area map with labeled survey points. This imagery layer was not included in the deliverables to the client as it required an Esri account and it was not part of the requested 
final product. However, the map was necessary to observe the greater area as the drone imagery did not cover the entire wetland area and there is some mismatching at the edges.

\subsection{Data Scrubbing and Loading}

The imagery layers were received through an online storage site as multiple zip files which were downloaded into a designated file in the $\mathrm{C}$ drive for sorting and backed up on a $1 \mathrm{~TB}$ storage device. All the files had to be unzipped one by one-including all the subfolders. There were two main folders-one for 2018 and one for 2019. Within the 2018 folder were the folders for the drone imagery and additional folders for orthophoto layers and individually flown nest sights — only the imagery from the drone folder was used. The 2019 file was organized differently. It had three subfolders including: June, July, and Orthophotos. Each of those subfolders had nine folders for individually processed datasets - including orthophotos, color NDVI, and Scientific NDVI datasets. Only the June 2019 dataset was used for this project.

This choice was necessary because, as stated before, the 2018 files did not include NDVI layers nor did the drone imagery have an infrared band to make a NDVI layer. The NDVI layers that were provided did not distinguish vegetation from water very well or at all for some areas. Once the necessary images were selected, they were loaded into mosaic raster sets in ArcMap where statistics and pyramids were created. The statistics and pyramids were necessary later to help improve the classification process.

The preparation for the bird survey data was straightforward. The first step was downloading the file from an email, then saving the file to the project folder containing the ArcMap file. The file was brought into the project, converted from a KML file into a point feature class and stored in the geodatabase containing the mosaic datasets from 2018 and 2019. The points were then projected to the same coordinate system as the raster layers. The change was necessary for the analysis later in the R-script - although there is a way to convert within the code as well if survey points used in the future are not re-projected ahead of time.

\subsection{Summary}

The database model for this MIP was straightforward conceptually and logically. This was because the client's deliverables did not need a very complex model or system. The requirements called for mosaic datasets, classified imagery, analysis results and point data. The data preparation process was straightforward as well. There was no need for an advanced query process or organization.

Difficult decisions were made to exclude the species identification and second 2019 dataset due to technical data considerations (no infrared and more spectral differences) and the extra months it would take to complete the incremental July 2019 classification.

Care was taken in creating layers that could be used by the client for future projects, an example being the feature layers and high-resolution classification.

The process of implementing this database design and the layer choices are discussed at length in the Chapter 5 . 


\section{Chapter 5 - Implementation}

Implementation is the "methods" part of this MIP and describes in detail how the project was executed -- from the creating of the multiple training sets to the interspersion calculation in R-Studio. The bulk of the time during this project was spent on classifying the imagery, primarily due to the spectral differences between one image layer to another within a mosaic and the fine resolution of the drone imagery. Section 5.1 expands on the process used to classify the images. Once the classification stage was completed, the classification layers were exported to a file for analysis in R. The full explanation for the R-Studio interspersion calculations are explained further in Section 5.2.

\subsection{Image Classification}

The classification portion of this project was straightforward in application. However, much time was needed to complete all the stages: sorting and applying definition queries to the mosaiced imagery, training, running the classification tool, error checking, and filtering. The first three stages were repeated until the resulting classification was adequate for the analysis steps outlined in section 5.2. The process described in this section details the process for the 2018 dataset. The same procedure was used on the June 2019 dataset.

To complete the first stage of the classification process, study area images from a single year were evaluated for spectral similarity and grouped accordingly. There were two queries in total, which selected the images with similar spectral characteristics. The images had to be grouped because of the differences in their spectral characteristics. This helped alleviate the issues stemming from the differences in data collection time mentioned in previous chapter.

The image names came from the attribute tables provided in the 2018 dataset. These labels are used throughout the Audubon data and are structured using an abbreviation of the site areas name-Wigwam Bay in this case (WIGW) — and a number between one and nine (01-09), indicating the study site. The sorting into two groups was necessary to improve the quality of the classification layers by grouping study areas with similar spectral characteristics.

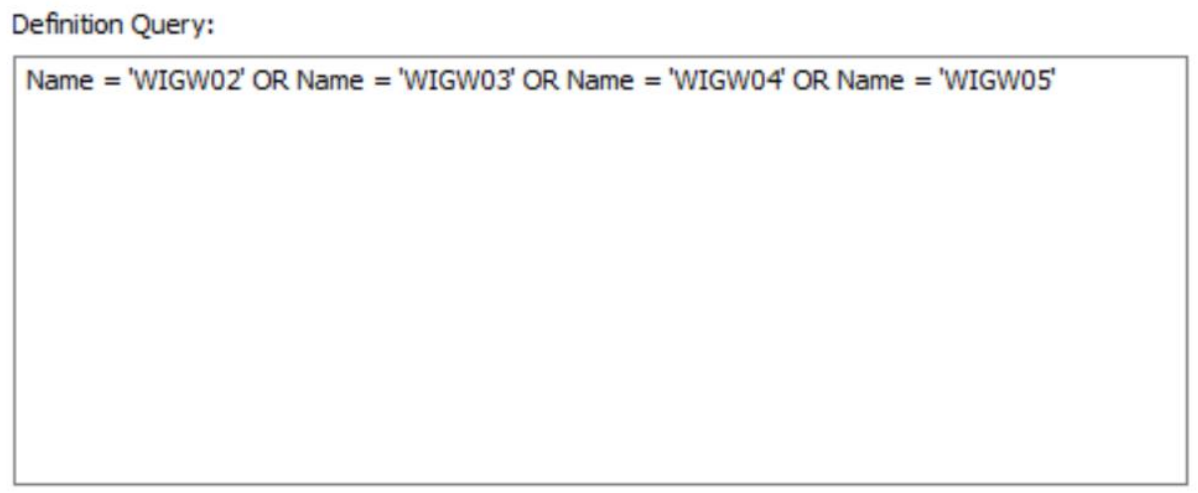

Query Builder...

Figure 5-1: Image Selection for Classification 
Once the sorting step was completed, the sampling or selection of training data from each set of images began. The samples were saved using the Training Sample Manager in the ArcGIS classification tool bar. The images selected using the query were used to create training samples shown in Figure 5-2. Inside the Training Manager, each class was named per the client's specifications and colors were assigned for each class: Open Water, Exposed Mud, Floating Vegetation, Emergent Vegetation, Trees / Shrubs, and Other.

\begin{tabular}{|c|c|c|c|c|}
\hline E回 & 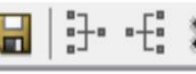 & $\downarrow \stackrel{1}{N}$ & 柾 & 回 \\
\hline ID & Class Name & Value & Color & Count \\
\hline 1 & Open Water & 1 & & 79530 \\
\hline 2 & Exposed Mud & 2 & & 25576 \\
\hline 3 & Floating Veg & 3 & & 167002 \\
\hline 4 & Emergent Veg & 4 & & 150466 \\
\hline 5 & Tree / Shrubs & 5 & & 88200 \\
\hline 6 & Other & 6 & & 27065 \\
\hline
\end{tabular}

Figure 5-2: 2018 Training Samples for Selected Images

Floating vegetation included the plants found on top of water bodies. The Emergent Vegetation class, the most observed, was comprised of grasses and plants. Woody shrubs and larger trees primarily found around the perimeter were classified as Trees/Shrubs. The "Other" class was composed of fallen logs, exposed trunks, and parked white vehiclesanything that did not fit into the previous four classes - though this class was only used in one of the classification training samples since these features were not near the study areas and therefore not included in the interspersion analysis. Trees/Shrubs were also not carried forward into the analysis as they were few and mostly outside the study areas.

The quality of the samples was tested using the "Interactive Classification" toolalso part of the Classification toolbar - which uses the same equation and function as the "Maximum Likelihood" tool conventionally used for supervised classification. The only difference between the approaches was that the Interactive tool did not require a signature file - which could not be created due to the sheer size of the samples needed to properly classify the imagery. The large sample sizes were necessary because even with the separation of the images, there was still spectral overlap between classes that made accurate sorting difficult - especially in spots with glare from a high sun.

In total there were about eight iterations per year of sample gathering and testing before the software produced classification layers that matched the source imagery well enough to be used in a landscape categorical based tool. This was checked visually, with an emphasis on the nine survey areas. While not scalable, the relatively small areas made this feasible. Figure 5-3 shows an overview of the 2018 classification and Figure 5-4 depicts the area shown in the red box below for Study Area 8. 


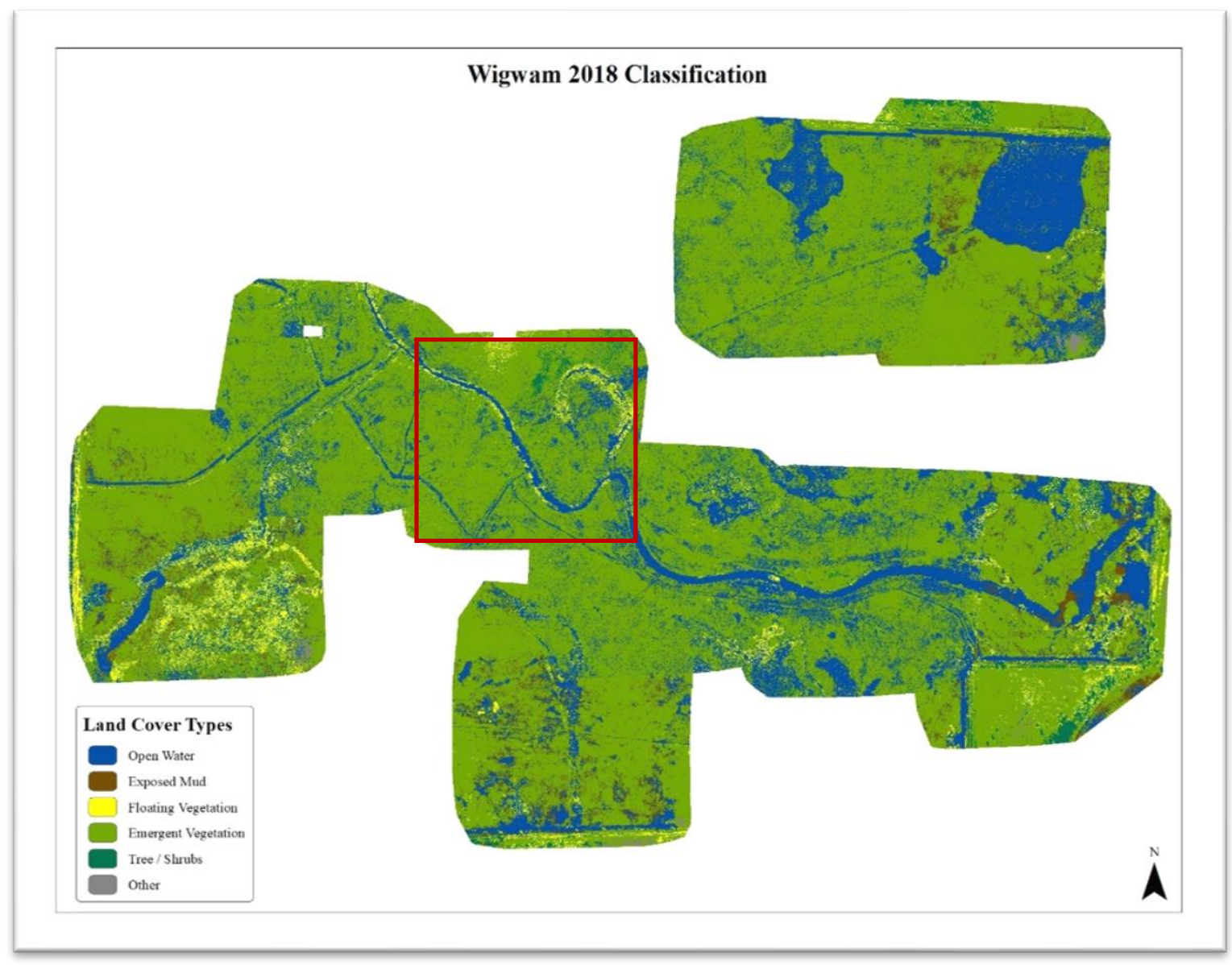

Figure 5-3: Wigwam Bay Wetland Study Area 2018 Classification Overview 


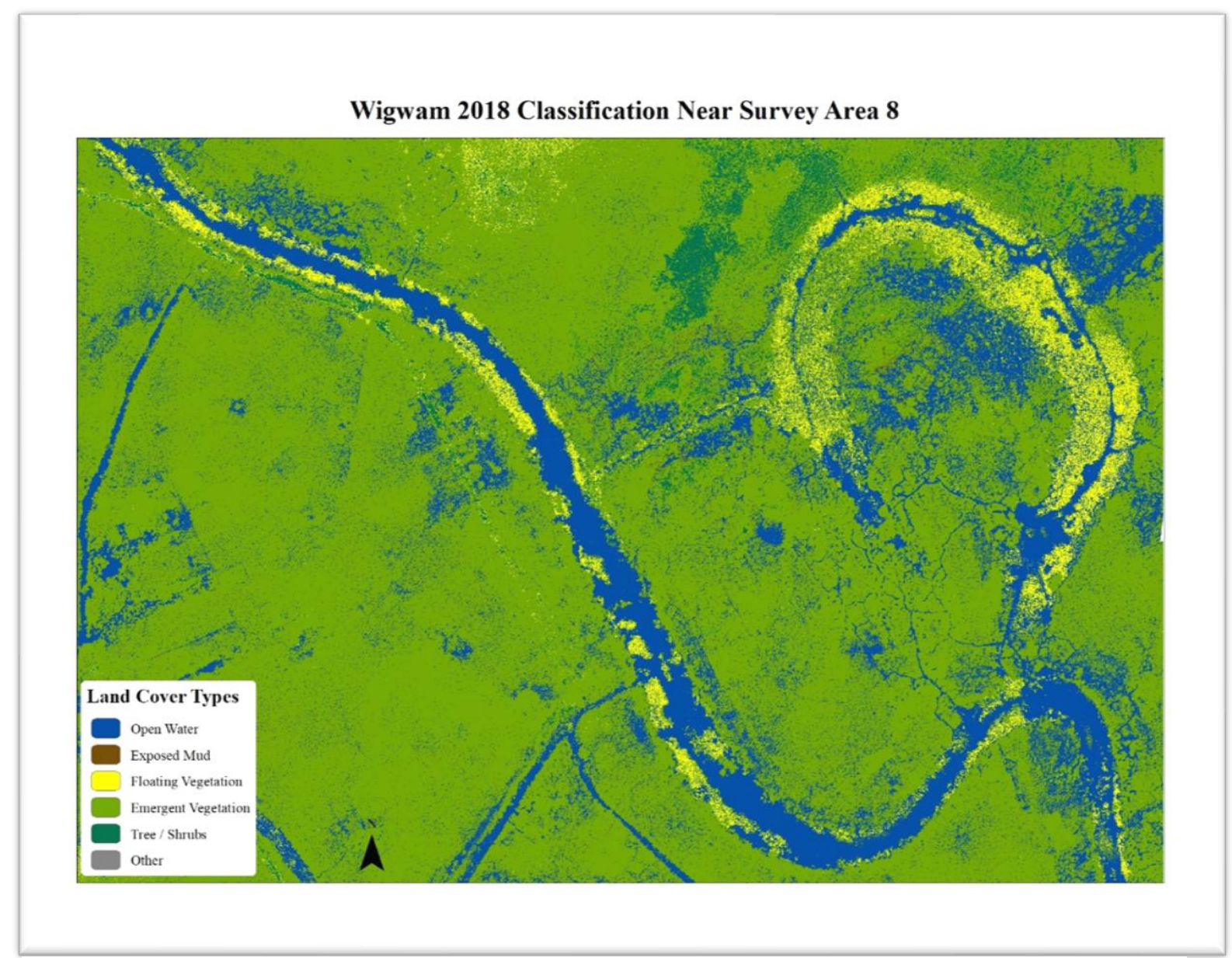

\section{Figure 5-4: Focused Classification Around Survey Point WGIW-08}

Once the classification was complete, the temporary classified layers created by the Interactive tool were saved to the geodatabase and merged to make one layer. Finally, the 2018 classification layer was reclassified to only include the four classes to be used in the interspersion analysis. Once this process was complete for 2018, the same techniques and processes were used on the June 2019 dataset.

After classification was complete for both 2018 and 2019, the classification layers were exported to GeoTiff format to a designated folder. The files were then used as input for the R-Script that was created to run and compile interspersion calculation and supporting metrics.

\subsection{Analysis using Landscapemetrics}

There are two commonly used software packages for quantifying landscape measurements including interspersion- "FRAGSTATS" and an open source alternative library used with R-studio, Landscapemetrics. This project used R-Studio with the library because FRAGSTAT software no longer accepts ArcGIS layers directly and the Audubon expressed a preference for re-usable R script and the Landscapemetrics library. The landscapemetrics library tools were used to calculate class and landscape interspersion 
metrics. The landscape metrics calculated in this analysis are part of the Interspersion and Juxtaposition Index (IJI), explained in detail in 2.2.

Two additional metrics, indirectly useful for interspersion were also calculated: Mean of Patch Area (AREA_MN) and Total Area (TA). These were added for insight into the interspersion metric results. At the landscape level, the Mean of Patch Area when used in combination with the Total Area metric can give an idea of patch structure, such as many small patches versus a few large patches. Because the interspersion metric uses patches as the basis for measurement, it was important to understand the size and area characteristics being used by the interspersion calculation. Knowing the structure is helpful because patch edges are used for the interspersion calculations. The total area metric also helped to confirm the study area sizes by summing all the areas at the patch level. Both area metrics are reported in hectares with the metrics' results ranging from 0 hectares without an upper limit (one hectare is equivalent to 10,000 square meters). 


\subsubsection{R-Studio}

The overall $\mathrm{R}$ analysis is shown in Figure 5-5 and a detailed description follows. The flowchart is broken into two main parts: computer and code preparation, followed by running the analysis tool. 


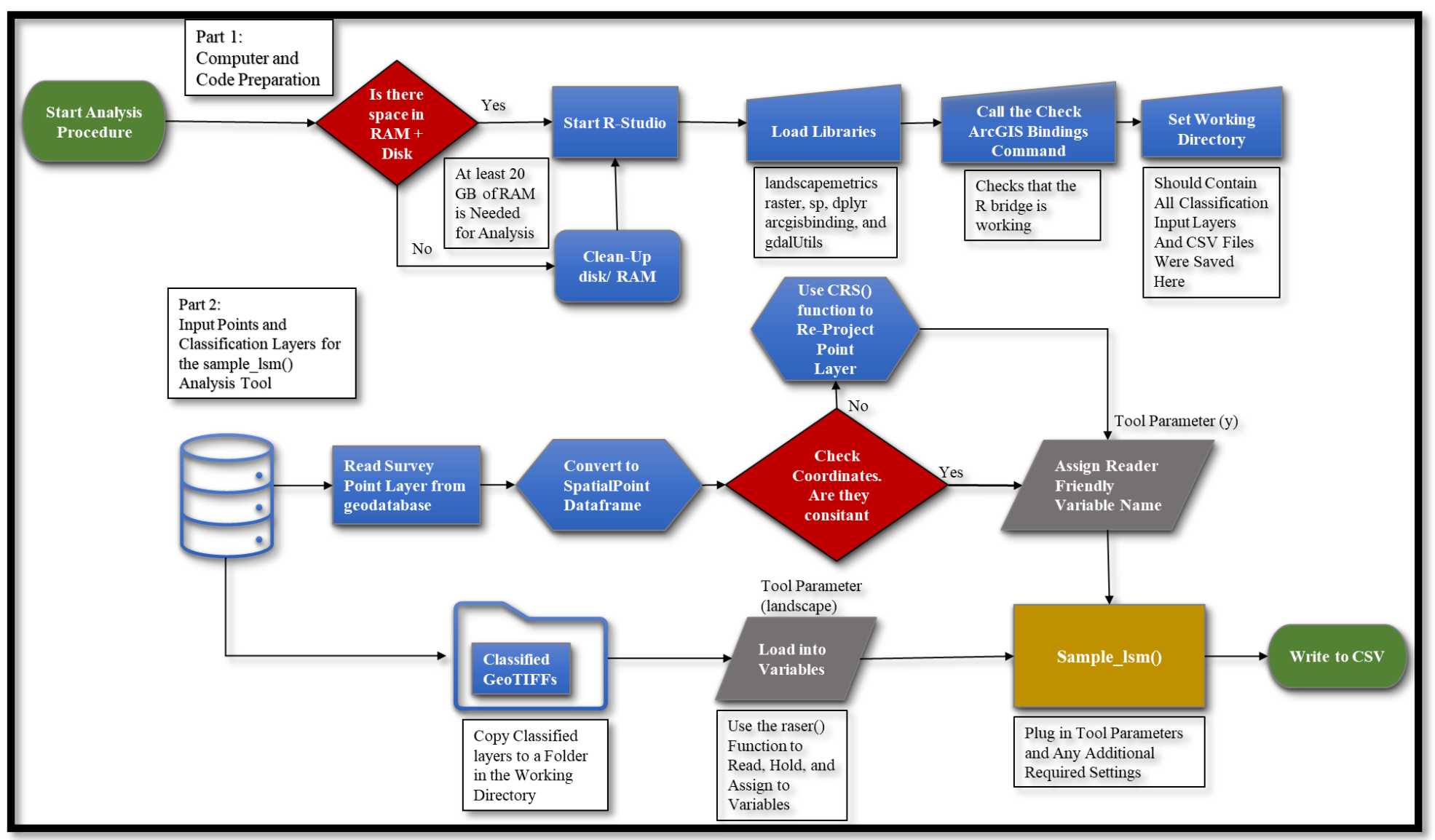

Figure 5-5: Full Analysis Workflow Diagram 
Part 1 is shown in Figure 5-6. The first step in the analysis portion of the project was to maximize the computer resources available to R-studio: cleaned up the geodatabase, freed up the maximum disk and memory, stopped all non-essential processes, and assigned all the memory to $\mathrm{R}$ manually with a built-in command and monitored using a task manager application. This first step is important because the process of running the analysis is very computationally intensive and uses much of the computer's memory, making memory management a critical success factor.

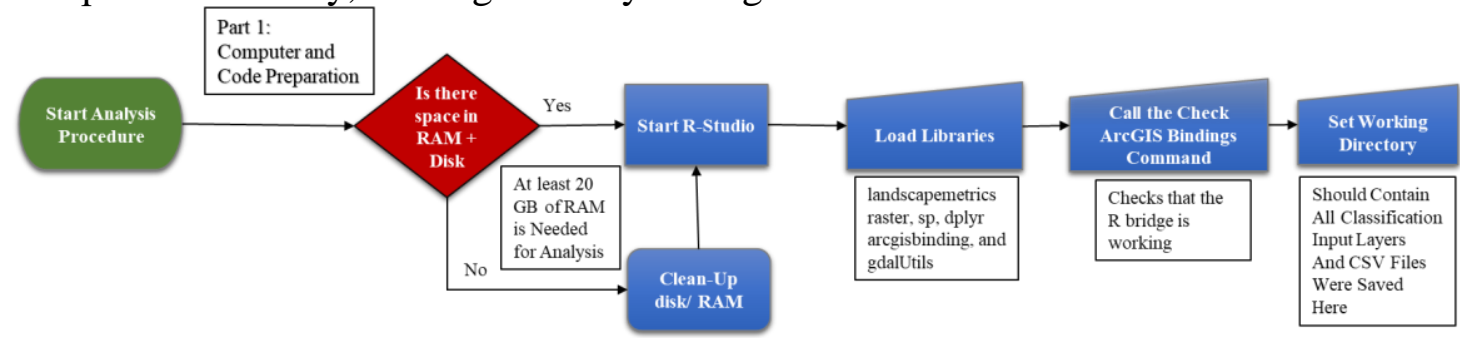

Figure 5-6: Part 1 of the Analysis Workflow

Once the computer was ready, the script loaded the necessary supporting libraries: raster, landscapemetrics, ArcGIS binding, gdalUtils, sp, and dplyr. Once those were loaded, the workspace or folder containing the desired input imagery - the two reclassified layers from 2018 and 2019.

Once the steps for Part 1 were completed the script moves on to the processing and analysis of input layers created previously in ArcMap shown in Figure 5-7.

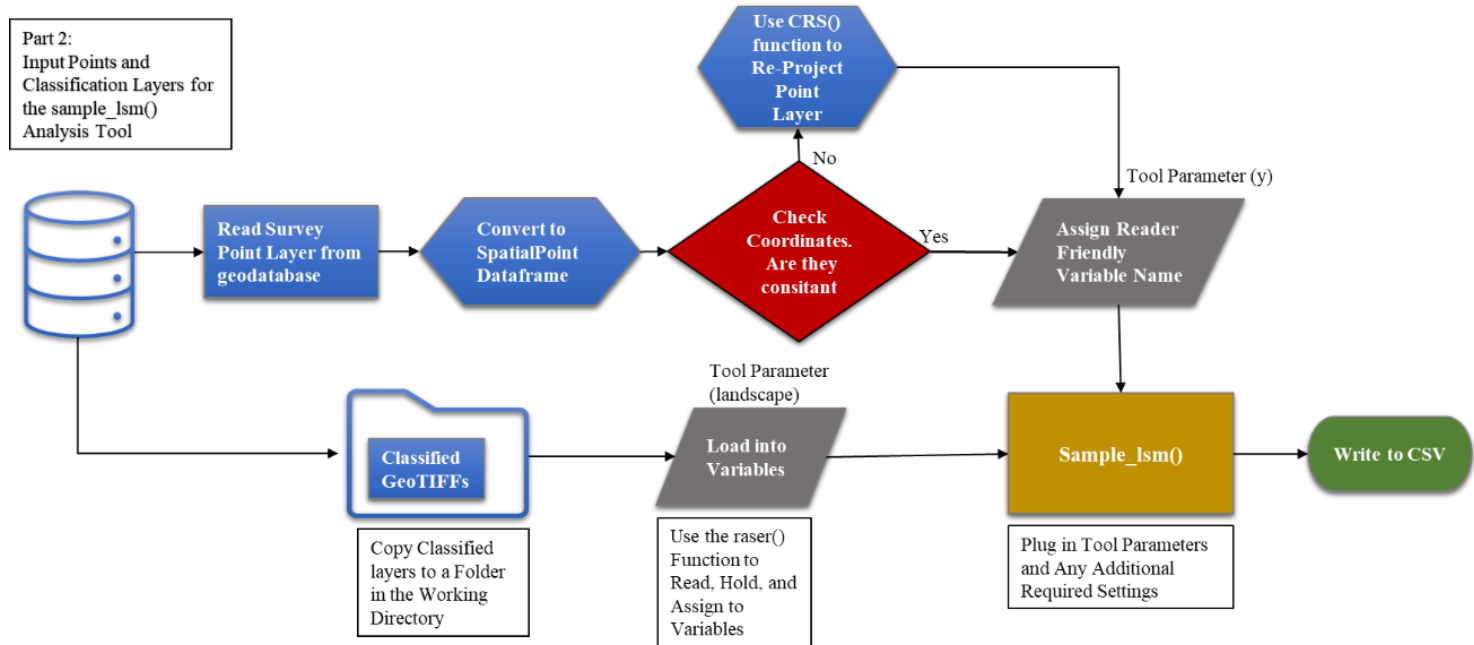

Figure 5-7: Part 2 of the Analysis Workflow

The ArcGIS raster layers were exported to Tiff before being loaded into the variable names. The next step was to open and convert the survey point layer to the appropriate Spatial Point data type using the sp and ArcGIS binding library tools.

After the points and raster layers were loaded, the survey points' coordinate system was checked visually to make sure all coordinate systems matched before the tool ran the metrics. If the point layer's coordinate system did not match, then it was reprojected to match the Tiff layer. 
To calculate the interspersion for the survey sites, the sample_lsm () function was used. The mandatory inputs for this project were the reclassified Tiff layers, survey points, radius size, metric names, and the desired shape of the buffer area. "Landscape" is the tool's placeholder name that the categorical raster layers are fed into automatically when parameters are filled in, "size" in this case is the radius of the desired sample area within the input imagery, "what" refers to the exact metrics to be calculated. The last parameter used for this analysis was "shape," which defines whether the sample clip will be a square or circle-square is the default. A sample code of using the function sample_lsm is in Appendix B and includes the use of a previously mentioned librarydplyr - to combine the results to one data frame to write the results to a CSV file smoother.

There is a caution to be aware of while using the sample_lsm function. The size of the actual sampled landscape can be different from the provided size. This can happen due to two main reasons. One, because clipping raster cells using a circle or a sample plot that is not exactly at the cell center can lead to inaccuracies. Another issue is that sample plots can exceed the landscape boundary. Because of this, included in the result table output is a column "percentage_inside" which refers to the actual clipped sample plot area in relation to the actual distance used in coding the function. For this project, all the percentage_inside values were at least $99.8 \%$.

The analysis for the 2019 data was repeated using the same code in a sequential manner. It was necessary to repeat steps to maximize the computer's resources before beginning. A detail to note about the sample_lsm() function as well as any others within the landscapemetrics library is that the default output is a "tibble" or an type of table viewable only in R-Studio, or publishable if using R-notebook. In this case the results were written directly to CSV files by year to be viewed, sorted, and saved more easily. The raw results from the calculation are in Appendix A and the full R-Script code can be found in Appendix B. For detailed analysis results with figures and charts, see Chapter 6.

\subsection{Summary}

This chapter detailed the steps taken to classify and then analyze drone imagery. The first section of this chapter focused on the supervised classification methods used and the preprocessing required. This included the formation of mosaic datasets, creating the definition queries such that only images with similar spectral characteristics were trained and classified at a time. The query step was important in this project because it helped to lessen error and mis-classification due to different land cover types having similar colorations. The classified images were then re-joined using a mosaic tool and reclassified to only include the land cover types to be used in the analysis step - in this case Open Water, Exposed Mud, Floating Vegetation, and Emergent Vegetation. Following the classification was a description and summary of the R-studio opensource library used to analyze the land cover types. The summary gave more detail on how the metrics and their outputs are structured and gives context for the two extra metrics added to the analysis. The metrics used from the library included: landscape level IJI, class level IJI, Mean Patch Area, and Total Area.

The tool used to execute these metrics was sample_lsm. This tool used the bird survey points from the project geodatabase to evaluate the chosen metrics within the specified radius of 150 meters. 
To help with the organization and exportation of the results, additional commands were used such as the two from the dypler library shown in the script excerpts. The raw results from the analysis output can be found in Appendix A. It should be noted that though the script for the analysis was simple and short, running the tools takes time and a large amount of RAM to complete. This is because much of processing for $\mathrm{R}$ - the engine behind the R-Studio user interface-runs mainly in memory and the larger the input dataset, the longer it will take to process. 


\section{Chapter 6 - Results and Analysis}

In this chapter, the results from the interspersion analysis are explored in detail as well as the aspects that worked and where problems were encountered. Section 6.1 talks about the findings and discusses what they mean in the context of the client's goals and how they can be used to implement conservation decisions. Sections 6.2 and 6.3 of this chapter answers questions about how the results and suggestions were useful to the client. Discussion on future work, steps to further improve this project, and future adjustments are detailed in Chapter 7, the last chapter of this report.

\subsection{Interspersion}

The calculated landscape metrics were written to CSV files by the R-script and then saved as Excel worksheets (.xlsx). There were two Excel files, one for each year with tabs containing the landscape and class level metrics for all nine study areas.

The purpose of the analysis for this functional deliverable was to find survey sites with reduced interspersion, indicating that landscape patches are becoming clumpier-a characteristic commonly seen with invasive plants since they tend to grow in bunches, out-competing native plants. Graphically and numerically, this means that if the Emergent Vegetation class level interspersion declines from year to year, it is a negative trend for habitat health, as is a low class level interspersion percentage. Conversely, increases in interspersion are a positive trend.

\subsubsection{Landscape Level Interspersion}

The landscape level interspersion results are shown below. This is the main set of results requested by the client. The highest interspersion is found in Study Area 5 and the lowest in Study Area 2. The overall interspersion change at the landscape level was minimal over the two-year period with eight out of nine sites improving, suggesting that the habitat is getting healthier. However, the changes calculated are very small and only cover two years, so while this is a positive baseline study, it will take more years to confirm the two-year positive trend. Most habitat studies span many years. 


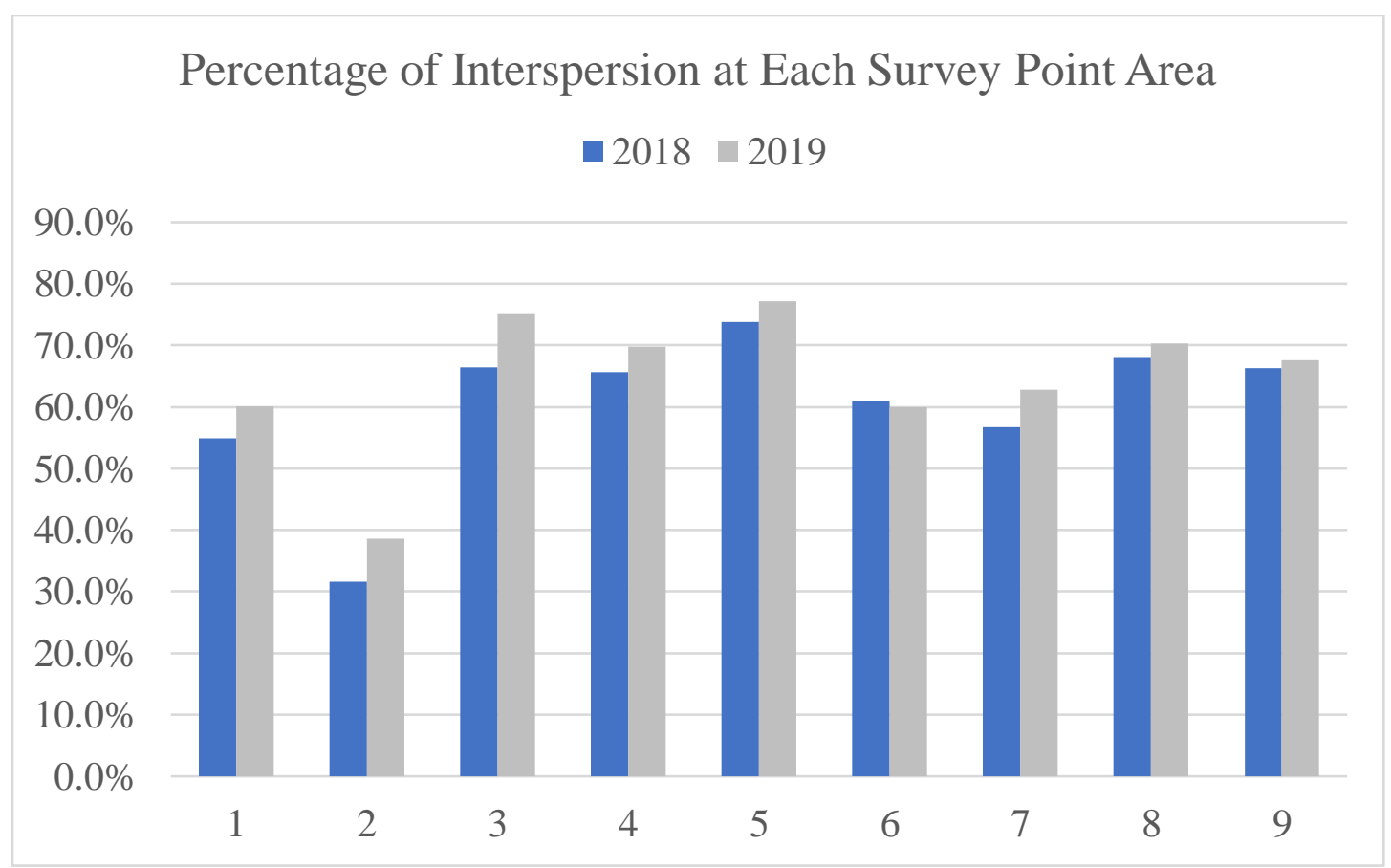

Figure 6-1: Landscape Level Interspersion Rate Change

The main finding at the landscape level is that Study Area 2 is clearly a priority for investigation as it is had the least interspersion at the landscape level. Study Area 2 is discussed in more detail below in the class metric results.

Only Study Area 6 showed a slight negative trend with a decrease in interspersion. On the classified imagery, shown in Figure 6-2, it can be observed that there is less Floating Vegetation in the central waterway in June 2019, therefore this change appears to be correct.
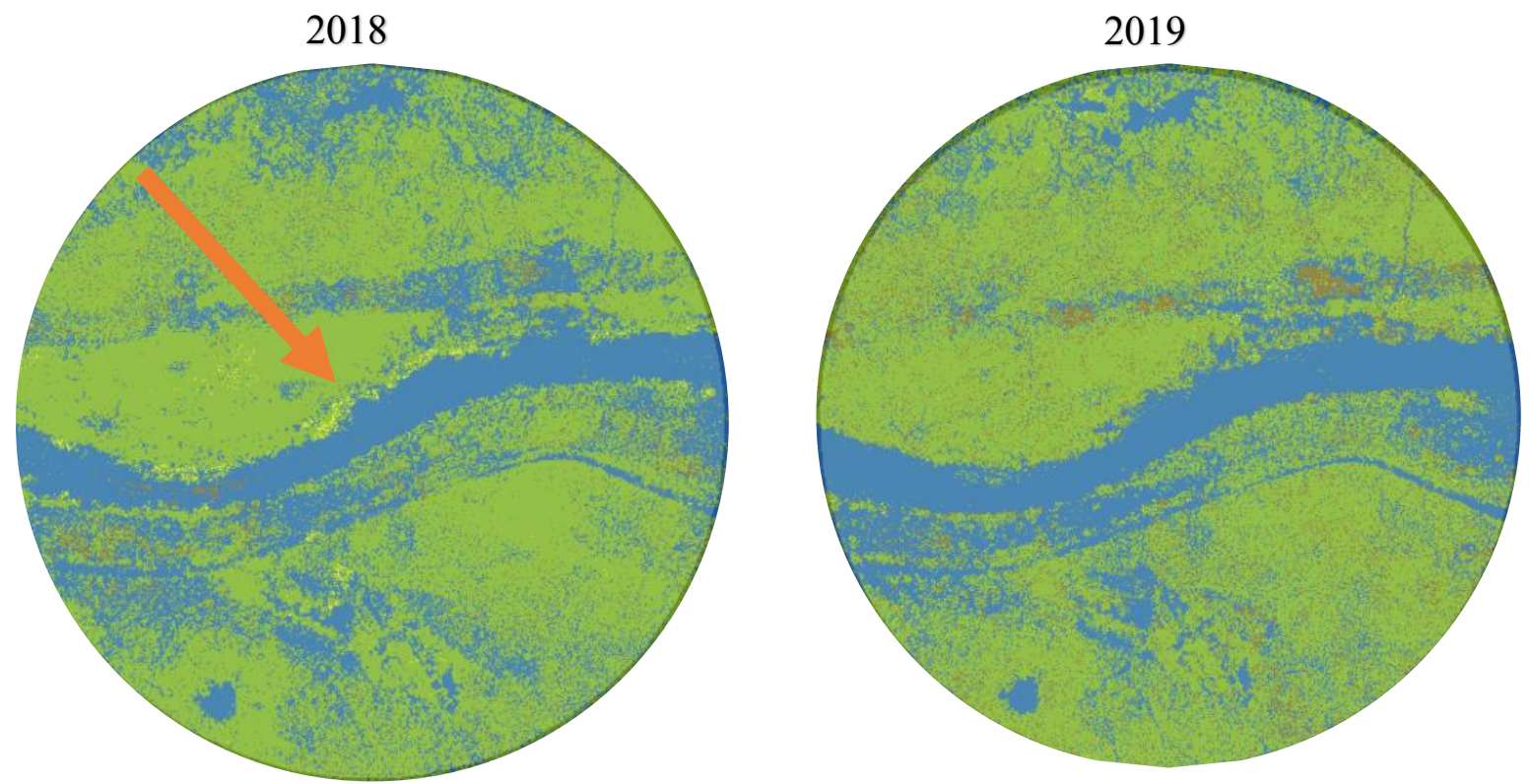

Figure 6-2: Study Area 6 Classification Change 
The decline at the landscape level is also confirmed by the class level results - the interspersion for floating vegetation, shown in Figure 6-3, was zero-meaning there was no floating vegetation detected. The change was most likely due to flowing currents pushing the vegetation further downstream outside of the designated survey buffer distance. However, the landscapemetrics library tools only evaluate whether an attribute or surface type is present or absent - they do not provide information about cause. Cause must be interpreted through a combination of field observations and tool analysis results.

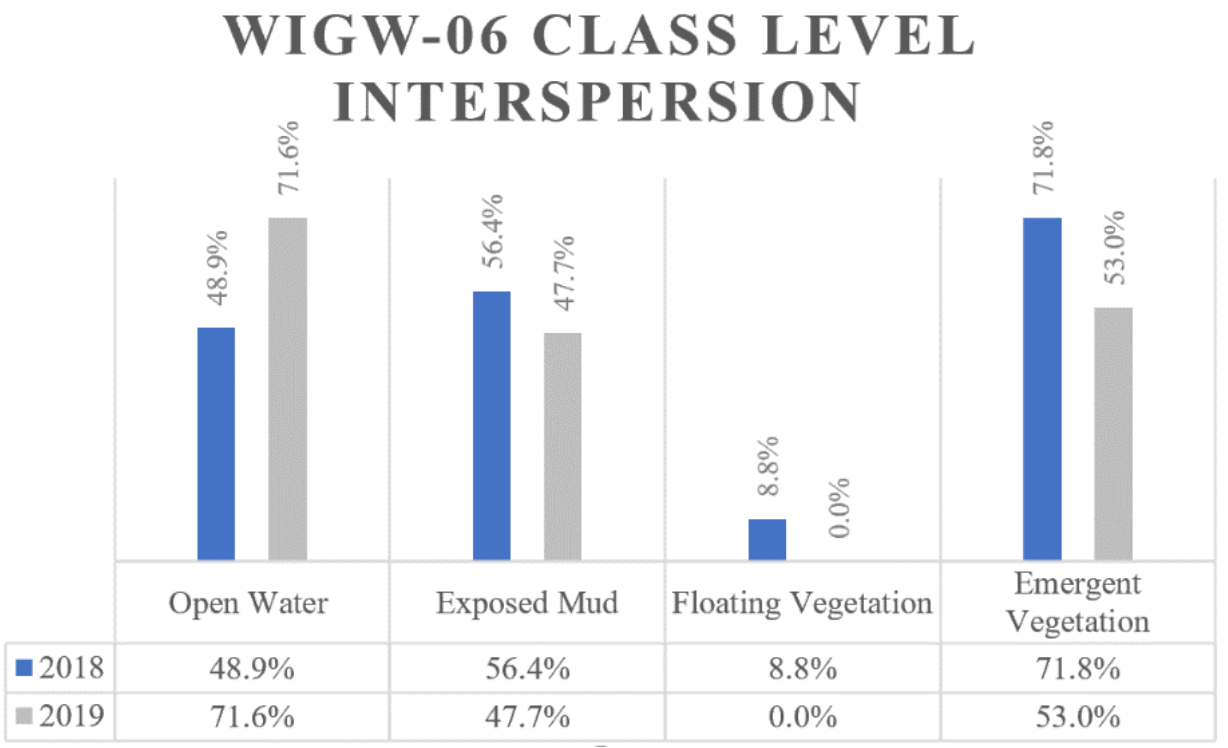

Figure 6-3: Study Area 6 Class Interspersion

\subsubsection{Class Level Interspersion}

While the above landscape level results are primarily positive, Emergent Vegetation Interspersion at the class level is decreasing at eight out of nine study areas, which suggests that invasive species are a concern and may be increasing in number despite the overall landscape level positive trend. At the class level, interspersion declines represent increased clumping, an indication of the presence of spreading invasive plants. Low metrics results for any single year may also suggest the presence of invasive plants.

The class metrics can be used to inform the prioritization of study areas for future investigation and possible mitigation. To create a preliminary prioritized list for further investigation and possible mitigation, the landscape level metrics was considered first and then class level Emergent Vegetation Interspersion metrics were used as a secondary consideration.

As the prioritization involves judgement, the nine sites were divided into high, medium, and low priorities for local site investigation and potential mitigation rather than an absolute ranking. The results are below in Table 4. 
Table 4. Priority Table

\begin{tabular}{|c|c|c|c|}
\hline Study Site \# & Level & Priority & Reason \\
\hline WIGW-02 & Landscape & High & $\begin{array}{l}\text { Lowest Interspersion: Range: } 31.7 \% \text { in } 2018 \text { to } \\
38.6 \% \text { in } 2019 \text {. }\end{array}$ \\
\hline WIGW-08 & Class & Medium & Largest decline: $89.9 \%$ to $46.82 \%$ (43.1 points) \\
\hline WIGW-09 & Class & Medium & $\begin{array}{l}\text { Second largest decline: } 85.0 \% \text { to } 44.0 \% \text { (41 } \\
\text { points) }\end{array}$ \\
\hline WIGW-05 & Class & Low & $\begin{array}{l}\text { Third largest decline: } 95.2 \% \text { to } 62.82 \%(31.4 \\
\text { points) }\end{array}$ \\
\hline WIGW-07 & Class & Low & $\begin{array}{l}\text { Fourth: Areas } 1,6,7 \text { had similar Interspersion } \\
\text { metric results at the landscape level (Range } 54.9 \% \\
\text { to } 62.8 \% \text { ), but site } 7 \text { has a decline of: } 68.4 \% \text { to } \\
42.9 \% \text { ( } 26 \text { points) }\end{array}$ \\
\hline WIGW-04 & Class & Low & $\begin{array}{l}\text { Fifth largest decline: } 86.0 \% \text { to } 63.1 \% \text { ( } 22.9 \\
\text { points) }\end{array}$ \\
\hline WIGW-06 & Class & Low & Sixth largest decline: $71.8 \%$ to $53 \%$ (18.8 points) \\
\hline WIGW-03 & Class & Low & $\begin{array}{l}\text { Seventh largest decline: } 79.3 \% \text { to } 61.29 \% \text { (18 } \\
\text { points) }\end{array}$ \\
\hline WIGW-01 & Class & Low & $\begin{array}{l}\text { Eighth largest decline: } 64.9 \% \text { to } 59.7 \% \text { (5.2 } \\
\text { points) }\end{array}$ \\
\hline
\end{tabular}

Study Areas 2, 8, and 4 are discussed below as examples of high, medium, and low prioritization.

Study Area 2 (WGIW-02) is a high priority due to its low landscape level interspersion. The class level results are shown in Figure 6-4.

\section{WIGW-02 CLASS LEVEL INTERSPERSION}

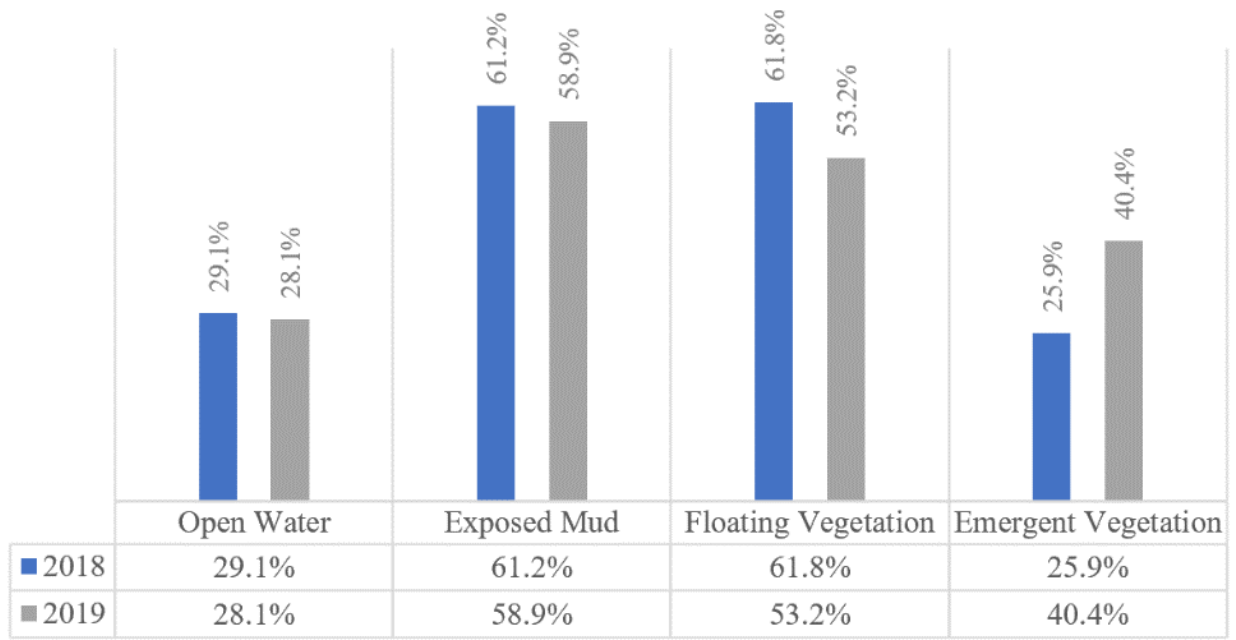

Figure 6-4: Study Area 2 Class Interspersion 
The Emergent Vegetation class, particularly in the 2018 dataset at the landscape level and 2019, showed very little interspersion (31.7\% to 38.6\%) which could indicate invasive species present in that area. With verification via source imagery, it was apparent that interspersion is very low in that survey area and that most of that region appears to be covered by a single type of emergent vegetation species. Although the class level metrics above in Figure 6-3 did show a year over year improvement, some of the improvement may be due to classification error because the two years do not appear very different in composition as shown in the Figure 6-5. Regardless, the low interspersion at landscape and class levels (25.9\% to $40.4 \%$ ) makes this a high priority for more investigaibs and possible mitigation.

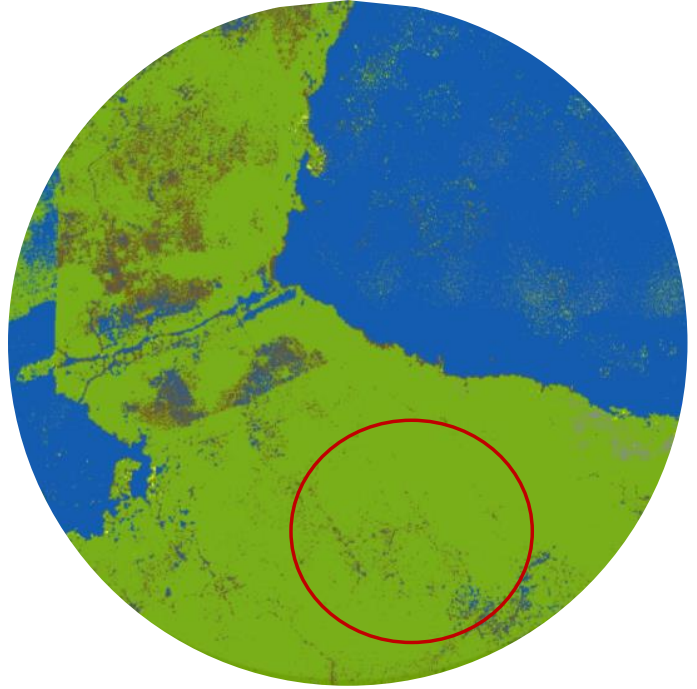

2019

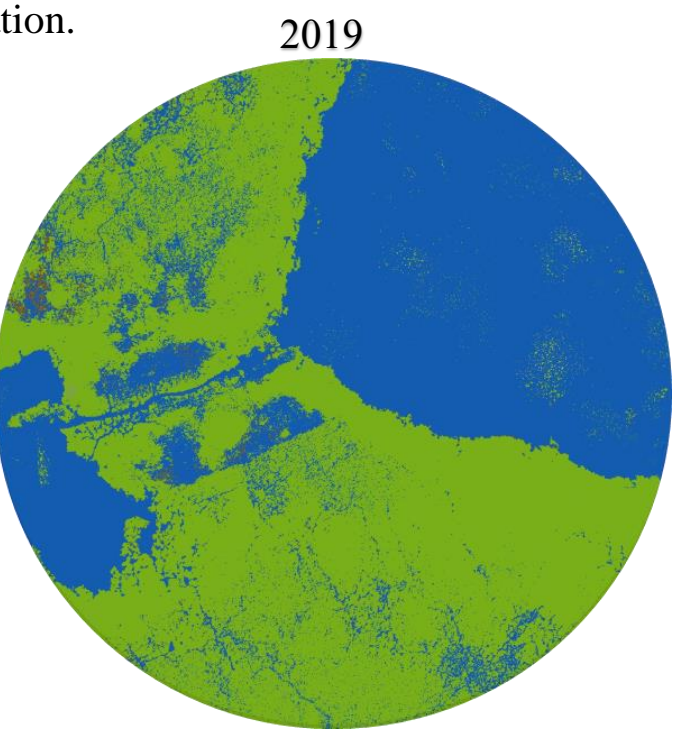

Figure 6-5: Study Area 2 Classification Change

WIGW-08, with results shown in Figure 6-6, had a significant decrease in Emergent Vegetation interspersion at the class level, with 43.1-point drop making this a medium priority. Visual confirmation using classification imagery in Figure 6-7 confirms some changes, but not as obviously as the 43-point drop would suggest. Due to the magnitude of the drop and the modest observed changes in the source imagery this is a medium priority. 


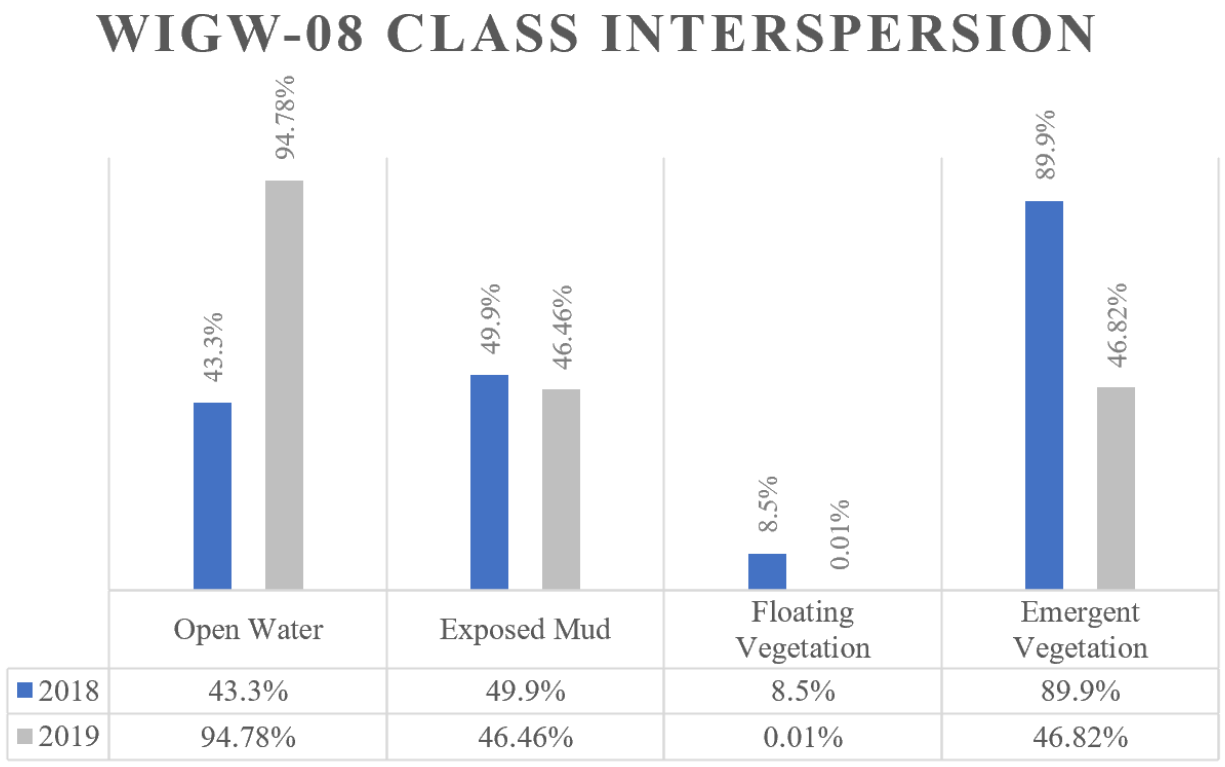

Figure 6-6: Study Area 8 Class Interspersion
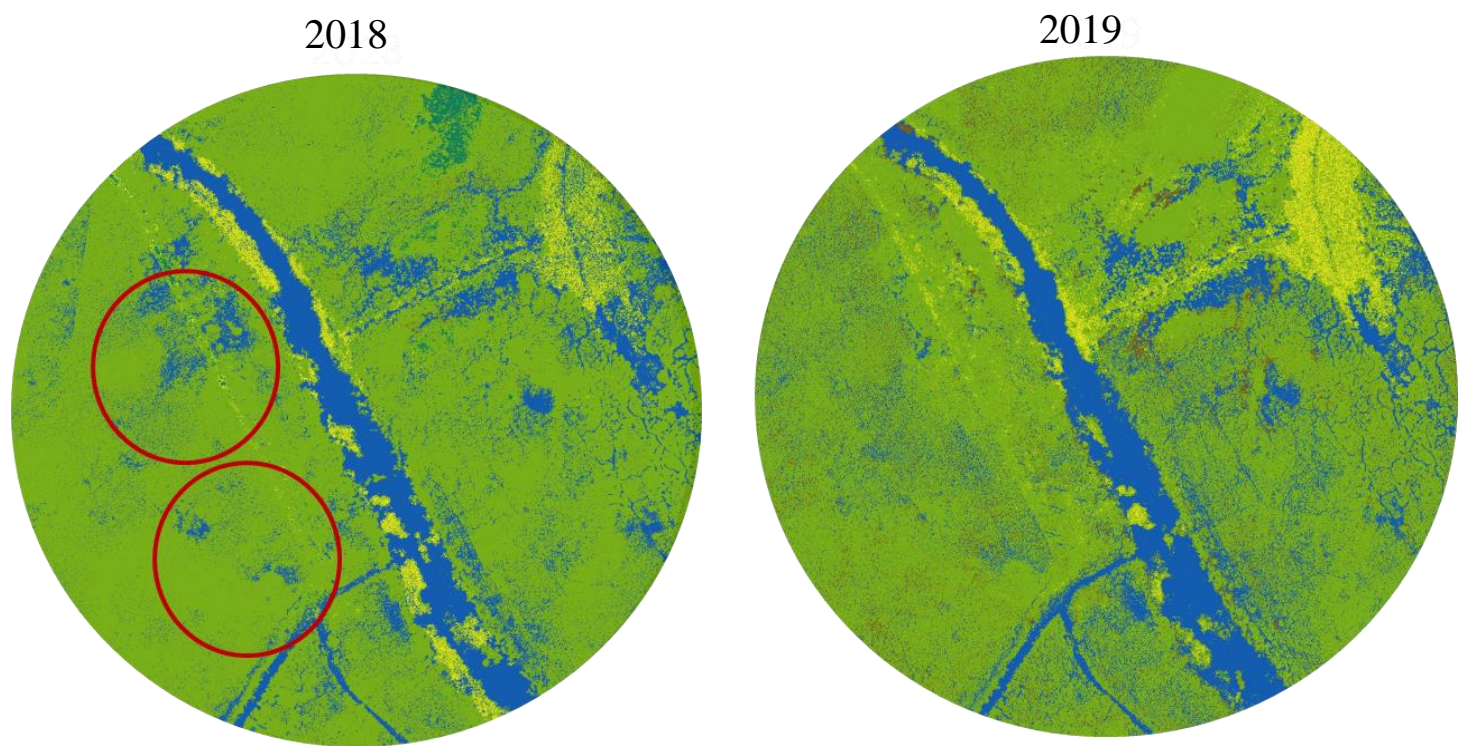

Figure 6-7: Study Area 8 Classification Change

WIGW-04 had a much less significant decrease in Emergent Vegetation interspersion, putting the study area in the low priority category with a 22.9-point drop in Figure 6-8. Visual confirmation using classification imagery in Figure 6-9 confirmed the similarity between years. 


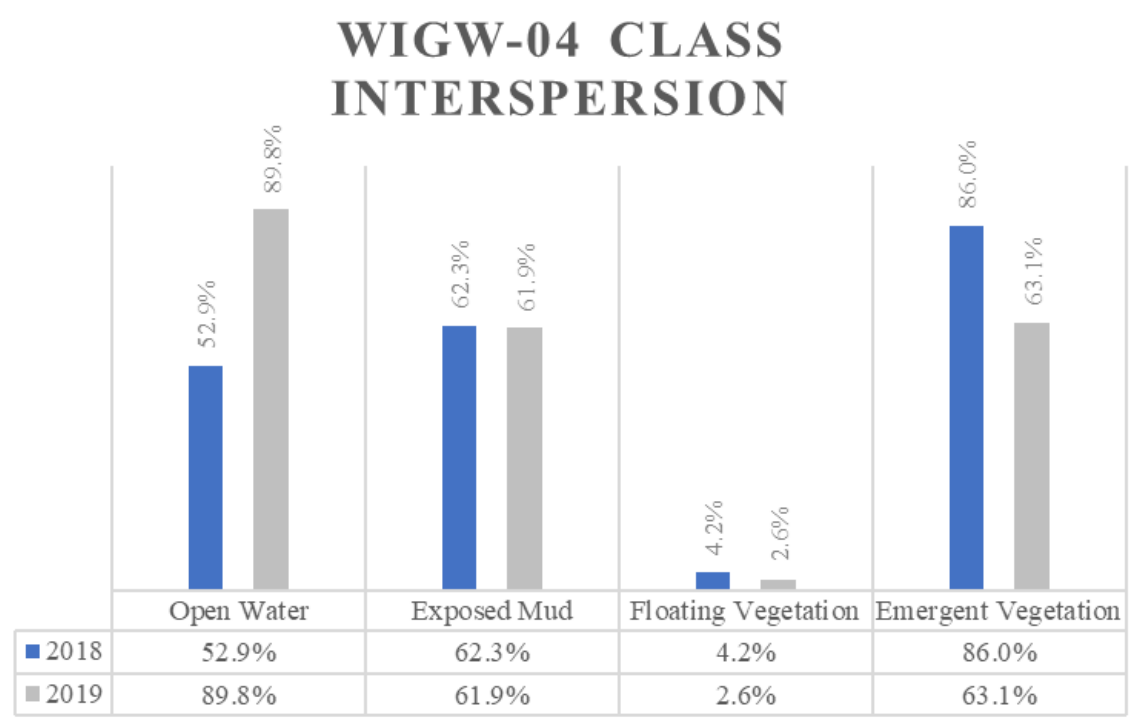

Figure 6-8: Survey Area 4 Class Interspersion
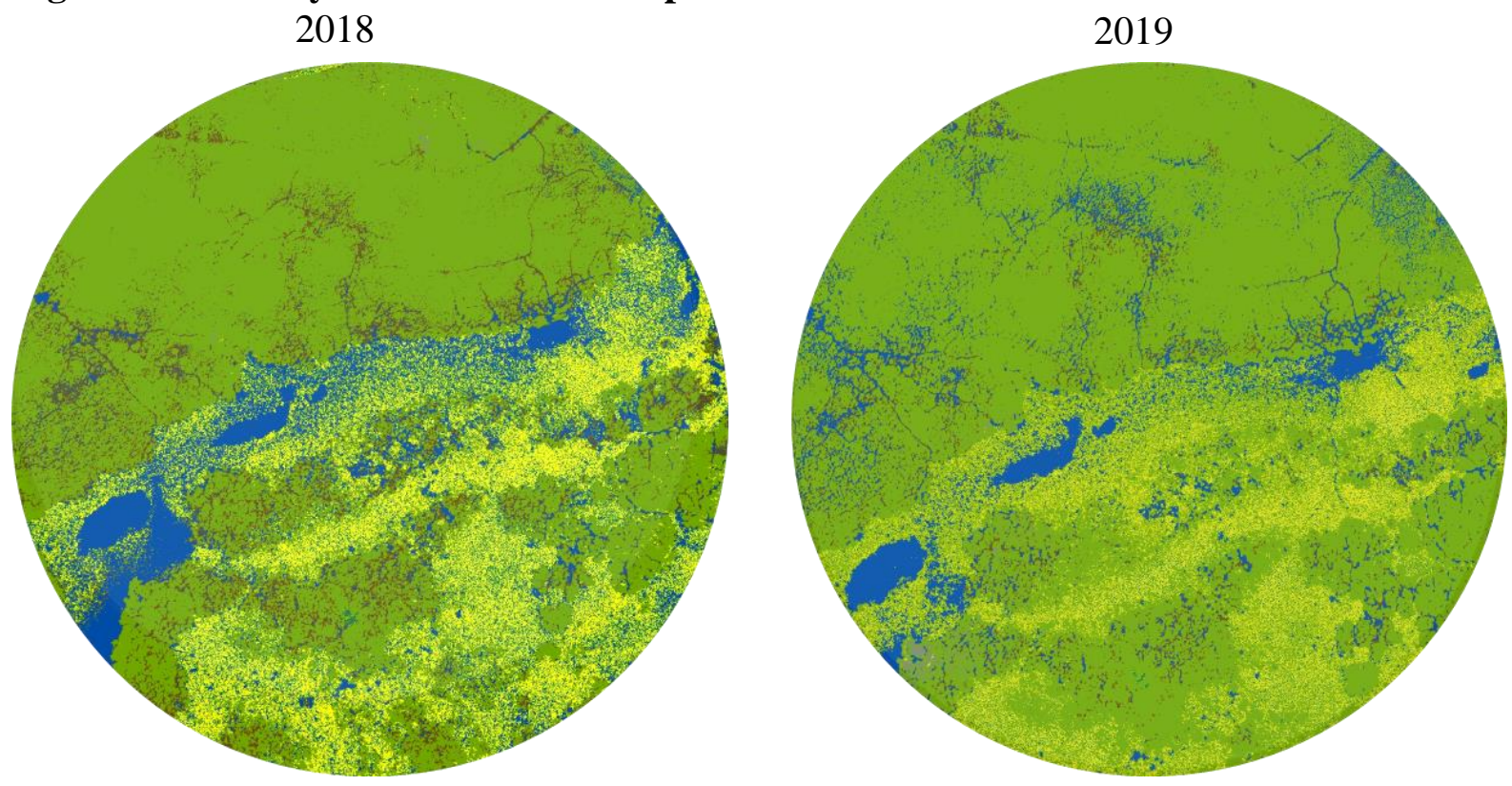

Figure 6-9: Study Area 4 Classification Change

Additional figures for the other survey areas can be found in Appendix $\mathrm{C}$ in the order ordered from high priority to low.

\subsection{Discussion}

The client asked for interspersion metrics at the landscape level - and these results appear accurate visually and positive for all but one of nine study areas. The class level results are not as positive and indicate that several study areas should be checked on site and assessed for mitigation and conservation, which is helpful for conservation efforts but not positive for habitat health in the immediate future. It is possible that some metric results are exaggerated in one direction or another, which was the reason to create the prioritized 
list using judgement categories to help the client make decisions regarding allocating conservation resources.

\subsection{Summary}

The analysis returned results showing that Survey Point 2 had the least interspersion out of the nine study areas. Because of this, it was given highest priority for on-site evaluation. The remaining sites were listed in priority order by Emergent Vegetation interspersion percent point decrease at the class level.

The resulting tables and charts were delivered to the Audubon Society for their assessment and reportedly will be taken into consideration for conservation planning. 


\section{Chapter 7 - Conclusions and Future Work}

In summary, the images and point layers provided by the client were stored in a database in mosaic datasets and a feature dataset, respectively. The mosaic dataset images were grouped according to color similarity before being classified into one of six category types. Once all images in a mosaic dataset were classified, the images were merged back into one layer per year. These merged classification layers were one of the client deliverables.

The classification layers for 2018 and 2019 were then reclassified down to four classes for analysis. These reclassified images were exported as GeoTiff layers and loaded into the R-Script provided in Appendix C. The script took in both classification layers as GeoTiffs and the bird survey points and produced two CSV files containing the metric results for each study area. The CSV files were saved as Excel Workbooks and metric results were compared to source imagery for verification and prioritization.

With the completion of this project, the functional and non-functional requirements specified by the client - the National Audubon Society - were fulfilled. Both 2018 and 2019 datasets were analyzed for interspersion and locations for further investigation were prioritized and presented in the Priority Table 4 shown in Chapter 6.

Suggestions for future work are to continue to work with the National Audubon Society to plan and execute drone flights for other wetland areas and use that opportunity to tailor the results from each data collection flight to better meet classification and analysis needs such as high-resolution imagery and timed flights. The analysis portion of the project may be sped up by utilizing the chunking option in R-Studio to break up larger raster layers into smaller, more manageable, pieces. Another option is to dedicate a project to identifying and mapping only the invasive species so that Audubon could obtain a more precise plan for conducting mitigation more strategically, possibly without local site visits. This might require using textural analysis with high resolution hyperspectral imagery, DEM models and well planned and executed drone flights. An extension could be to collect bird data as the beginning of a long-term project to evaluate correlation between marsh bird movement and land cover change. Finally, one study in Chapter 2 mentioned that water depth was important. If the Audubon believes this is significant for the Wigwam Bay Wetland Area, then this could also be added to the analysis for improved prioritization. 


\section{Works Cited}

Bellis, L. M., Pidgeon, A. M., Radeloff, V. C., St-Louis, V., Navarro, J. L., \& Martella, M. B. (2008). Modeling habitat suitability for greater rheas based on satellite image texture. Ecological Applications, 18(8), 1956-1966. doi:10.1890/07-0243.1

Burnham, K. P., Anderson, D. R., \& Hurvaert, K. P. (2011, January). AIC model selection and multimodel inference in behavioral ecology: some background, observations, and comparisons. Behavioral Ecology and Sociobiology, 65(1), 2335. doi: 0.1007/s00265-010-1029-6

Chambers, R. M., Meyerson, L. A., \& Saltonstall, K. (1999). Expansion of Phragmites australis into tidal wetlands of North America. Aquatic Botany, 64, 261-273.

Conway, C. J. (2011, September 1). Standardized North American marsh bird monitoring protocol. Wetlands, 34(3), 319-346. doi:10.1675/063.034.0307

Correll, D. M., Hantson, W., Hodgman, T. P., Cline, B. B., Elphick, C. S., Shriver, G. W., . . Olsen, J. B. (2019). Fine-scale mapping of coastal plant communities in the northeastern USA. Wetlands, 39(1), 17-28. doi:10.007/s13157-018-1028-3

Elhadi, A., Mutanga, O., \& Rugege, D. (2010). Mutlispectral and hyperspectral remote sensing for identification and mapping of wetlands vegetation: a review. Wetlands Ecol Management, 18, 281-296.

Gagnon Lupien, N., Gauthier, G., \& Lavoie, C. (2014, May 28). Effect of the invasive common reed on the abundance, richness and diversity of birds in freshwater marshes. Animal Conservation, 18(1), 32-43.

Gilsson, W. J., Brady, R. S., Paulios, A. T., Jacobi, S. K., \& Larkin, D. J. (2015). Sensitivity of secretive marsh birds to vegetation condition in natural and restored wetlands in Wisconsin. The Journal of Wildlife Management, 79(7), 1101-1116.

Gottschalk, T. K., Huettmann, F., \& Ehlers, M. (2005). Thirty years of analyzing and modelling avian habitat relationships using satellite imagery data: a review. International Journal of Remote Sensing, 26(12), 2631-2656.

Hanson, H. C. (1975). The use of basic principles in the classification of range vegetation. Rangeland Ecology \& Management/Journal of Range Management Archives, 10(1), 26-33.

Haralick, R. M., Shanmugam, K., \& Distein, I. (1973). Textural features for image classification. IEEE Transactions on Systems, Man, and Cybernetics, 6, 610-621.

Hepinstall, J. A., \& Sader, S. A. (1997). Using Bayesian statistics, Thematic Mapper satellite imagery, and breeding bird survey data to model bird species probability of occurence in Maine. Photogrammetric Engneering and Remote Sensing, 63(10), 1231-1236.

Hesselbarth, M. H., Sciaini., M., With, K. A., Wiegand, K., \& Nowosad, J. (2019). Landscapemetrics: an open-source R tool to calculate landscape metrics. Ecography, 42, 1648-1657 (ver 1.4.5).

Kettenring, K. M., Mock, K. E., Zaman, B., \& McKee, M. (2016). Life on the edge: reproductive mode and rate of invasive Pragmites australis patch expansion. Biological Invasions, 18, 2475-2495.

Klemas, V. (2011). Remote sensing of wetlands: case studies comparing practical techniques. Journal of Coastal Research, 27(3), 418-426. 
Laba, M., Downs, R., Smith, S., Welsh, S., Neider, C., White, S., . . Baveye, P. (2008). Mapping invasive plants in the Hudson River National Estuarine Research Reserve using quickbird satellite imagery. Remote Sensing of Environment, 112, 286-300.

Liu, C., Jiang, H., Hou, Y., Zhang, S., Su, L., Li, X., . . Zhaofei, W. (2010). Habiitat changes for breeding waterbirds in Yancheng National Nature Reserve, China: a remote sensing study. Wetlands, 30, 879-888.

Marcaccio, J. V., Markle, C. E., \& Chow-Fraser, P. (2016). Use of fixed wing and multirotor unmanned aeriel vehicles to map dynamic changes in a freshwater marsh. Journal of Unmanned Vehicle Systems, 193-202.

McGarigal, K. (2015, April 21). FRAGSTATS help. Retrieved from UMASS Landscape Ecology Lab:

https://www.umass.edu/landeco/research/fragstats/documents/fragstats.help.4.2.p df

McGarigal, K., \& Marks, B. J. (1995). FRAGSTATS: Spatial pattern analysis program for quantifying landscape structure. Portland: U.S. Department of Agriculture, Forest Service.

Mutangs, A. O., \& Rugege, D. (2010). Multispectral and hyperspectral remote sensing for identification and mapping of wetland vegetation: a review. Wetland Ecology and Management, 18(3), 281-296.

Pengra, B. W., Johnston, C. A., \& Loveland, T. R. (2007). Mapping an invasive plant, Phragmites australis, in coastal wetlands using the EO-1 Hyperion hyperspectral sensor. Remote Sensing of Environment, 108, 74-81.

Philipp, K. R., \& Field, R. T. (2005, September 20). Phragmites australis expansion in Delaware Bay salt marshes. Ecological Engineering, 25(3), 275-291.

Pickens, B. A., \& King, S. L. (2014, August 10). Linking multi-temporal satellite imagery to coastal wetland dynamics and bird distribution. Ecological Modelling, 285, 1-12.

Rehm, E. M., \& Baldassarre, G. A. (2007, December). The influence of interspersion on marsh bird abundance in New York. The Wilson Journal of Ornithology, 119, 648-654.

Rosso, P., Ustin, S., \& Hasings, A. (2005). Mapping marshland vegetation of San Francisco Bay, California, using hyperspectral data. International Journal of Remote Sensing, 26(23), 5169-5191.

Samiappan, S., Turnage, G., Hathcock, L., Casagrande, L., Preston, S., \& Moorhead, R. (2017). Using unmanned aerial vehicles for high-resolution remote sensing to map invasive Phragmites australis in coastal wetlands. International Journal of Remote Sensing, 38(8-10), 2199-2217.

Scarbrough, K. A., Bourgeau-Chavez, L., Kowalski, K. P., Carlson Mazur, M. L., Powell, R. B., Brooks, C., . . . Riodan, K. (2013). Mapping invasive Phragmites australis in the coastal Great Lakes with ALOS PALSAR satellite imagery for decision support. Journal of Great Lakes Research, 39(1), 65-77.

Sciaini, M., Hasselbarth, M. H., With, K. A., Wiegand, K., \& Nowosad, J. (2019). Landscapemetrics: an open-source R tool to calculate landscape metrics. Ecography, 42, 1648-1657(ver. 1.45). 
The National Audubon Society. (2020, July 19). About Us. Retrieved from Audubon: https://www.audubon.org/about

Tozer, D. C. (2016, Febuary). Marsh bird occupancy dynamics, trends, and conservation in the southern Great Lakes basin: 1996 to 2013. Journal of the Great Lakes Research, 42(1), 136-145.

Xie, Y., Sha, Z., \& Yu, M. (2008, March). Remote sensing imagery in vegetation mapping: a review. Journal of Plant Ecology, 1(1), 9-23.

Xie, Y., Zhang, A., \& Welsh, W. (2015, January). Mapping wetlands and Phragmites using publically available remotely sensed images. Photogrammatic Engineering \& Remote Sensing, 81(1), 69-78. 


\section{Appendix A. Raw Results}

Table 5. Raw results from 2018

\begin{tabular}{|c|c|c|c|c|c|c|c|c|}
\hline & layer & level & class & $i d$ & metric & value & plot_id & percentage_inside \\
\hline 1 & 1 & class & 1 & NA & $\mathrm{iji}$ & 46.47005 & 1 & 99.97515 \\
\hline 2 & 1 & class & 2 & NA & $\mathrm{iji}$ & 42.9819 & 1 & 99.97515 \\
\hline 3 & 1 & class & 3 & NA & $\mathrm{iji}$ & 62.95777 & 1 & 99.97515 \\
\hline 4 & 1 & class & 4 & NA & $\mathrm{iji}$ & 64.91638 & 1 & 99.97515 \\
\hline 5 & 1 & landscape & NA & NA & $\mathrm{iji}$ & 54.91605 & 1 & 99.97515 \\
\hline 6 & 1 & class & 1 & NA & $\mathrm{iji}$ & 29.07021 & 2 & 99.99774 \\
\hline 7 & 1 & class & 2 & NA & iji & 61.23249 & 2 & 99.99774 \\
\hline 8 & 1 & class & 3 & NA & $\mathrm{iji}$ & 61.78249 & 2 & 99.99774 \\
\hline 9 & 1 & class & 4 & NA & $\mathrm{iji}$ & 25.87393 & 2 & 99.99774 \\
\hline 10 & 1 & landscape & NA & NA & $\mathrm{iji}$ & 31.67742 & 2 & 99.99774 \\
\hline 11 & 1 & class & 1 & NA & $\mathrm{iji}$ & 51.11344 & 3 & 99.61975 \\
\hline 12 & 1 & class & 2 & NA & $\mathrm{iji}$ & 62.60418 & 3 & 99.61975 \\
\hline 13 & 1 & class & 3 & NA & $\mathrm{iji}$ & 13.48656 & 3 & 99.61975 \\
\hline 14 & 1 & class & 4 & NA & $\mathrm{iji}$ & 79.3447 & 3 & 99.61975 \\
\hline 15 & 1 & landscape & NA & NA & iji & 66.40958 & 3 & 99.61975 \\
\hline 16 & 1 & class & 1 & NA & $\mathrm{iji}$ & 52.86291 & 4 & 98.64792 \\
\hline 17 & 1 & class & 2 & NA & $\mathrm{iji}$ & 62.3264 & 4 & 98.64792 \\
\hline 18 & 1 & class & 3 & NA & $\mathrm{iji}$ & 4.233779 & 4 & 98.64792 \\
\hline 19 & 1 & class & 4 & NA & $\mathrm{iji}$ & 86.01004 & 4 & 98.64792 \\
\hline 20 & 1 & landscape & NA & NA & $\mathrm{iji}$ & 65.62754 & 4 & 98.64792 \\
\hline 21 & 1 & class & 1 & NA & $\mathrm{iji}$ & 58.45042 & 5 & 98.0358 \\
\hline 22 & 1 & class & 2 & NA & $\mathrm{iji}$ & 62.67667 & 5 & 98.0358 \\
\hline 23 & 1 & class & 3 & NA & iji & 10.5695 & 5 & 98.0358 \\
\hline 24 & 1 & class & 4 & NA & iji & 94.17253 & 5 & 98.0358 \\
\hline 25 & 1 & landscape & NA & NA & $\mathrm{iji}$ & 73.76058 & 5 & 98.0358 \\
\hline 26 & 1 & class & 1 & NA & iji & 48.88175 & 6 & 99.3891 \\
\hline 27 & 1 & class & 2 & NA & $\mathrm{iji}$ & 56.43748 & 6 & 99.3891 \\
\hline 28 & 1 & class & 3 & NA & $\mathrm{iji}$ & 8.786315 & 6 & 99.3891 \\
\hline 29 & 1 & class & 4 & NA & $\mathrm{iji}$ & 71.77506 & 6 & 99.3891 \\
\hline 30 & 1 & landscape & NA & NA & $\mathrm{iji}$ & 60.9677 & 6 & 99.3891 \\
\hline 31 & 1 & class & 1 & NA & $\mathrm{iji}$ & 42.62573 & 7 & 99.72645 \\
\hline 32 & 1 & class & 2 & NA & iji & 47.89219 & 7 & 99.72645 \\
\hline 33 & 1 & class & 3 & NA & $\mathrm{iji}$ & 32.75627 & 7 & 99.72645 \\
\hline 34 & 1 & class & 4 & NA & iji & 68.37909 & 7 & 99.72645 \\
\hline 35 & 1 & landscape & NA & NA & $\mathrm{iji}$ & 56.76117 & 7 & 99.72645 \\
\hline 36 & 1 & class & 1 & NA & $\mathrm{iji}$ & 43.34188 & 8 & 97.71081 \\
\hline 37 & 1 & class & 2 & NA & iji & 49.93188 & 8 & 97.71081 \\
\hline 38 & 1 & class & 3 & NA & $\mathrm{iji}$ & 8.483679 & 8 & 97.71081 \\
\hline 39 & 1 & class & 4 & NA & $\mathrm{iji}$ & 89.92142 & 8 & 97.71081 \\
\hline
\end{tabular}




\begin{tabular}{|c|c|c|c|c|c|c|c|c|}
\hline 40 & 1 & landscape & NA & NA & $\mathrm{iji}$ & 68.15949 & 8 & 97.71081 \\
\hline 41 & 1 & class & 1 & NA & iji & 46.4152 & 9 & 99.0357 \\
\hline 42 & 1 & class & 2 & NA & iji & 44.07967 & 9 & 99.0357 \\
\hline 43 & 1 & class & 3 & NA & $\mathrm{iji}$ & 29.16099 & 9 & 99.0357 \\
\hline 44 & 1 & class & 4 & NA & $\mathrm{iji}$ & 85.00399 & 9 & 99.0357 \\
\hline 45 & 1 & landscape & NA & NA & $\mathrm{iji}$ & 66.27235 & 9 & 99.0357 \\
\hline 46 & 1 & landscape & NA & NA & area_mn & 8.37E-06 & 1 & 99.97515 \\
\hline 47 & 1 & landscape & NA & NA & ta & 7.062084 & 1 & 99.97515 \\
\hline 48 & 1 & landscape & NA & NA & area_mn & $5.42 \mathrm{E}-05$ & 2 & 99.99774 \\
\hline 49 & 1 & landscape & NA & NA & ta & 7.06368 & 2 & 99.99774 \\
\hline 50 & 1 & landscape & NA & NA & area_mn & $3.19 \mathrm{E}-05$ & 3 & 99.61975 \\
\hline 51 & 1 & landscape & NA & NA & ta & 7.036979 & 3 & 99.61975 \\
\hline 52 & 1 & landscape & NA & NA & area_mn & $3.30 \mathrm{E}-05$ & 4 & 98.64792 \\
\hline 53 & 1 & landscape & NA & NA & ta & 6.96833 & 4 & 98.64792 \\
\hline 54 & 1 & landscape & NA & NA & area_mn & 7.71E-05 & 5 & 98.0358 \\
\hline 55 & 1 & landscape & NA & NA & ta & 6.925091 & 5 & 98.0358 \\
\hline 56 & 1 & landscape & NA & NA & area_mn & $1.05 \mathrm{E}-05$ & 6 & 99.3891 \\
\hline 57 & 1 & landscape & NA & NA & ta & 7.020686 & 6 & 99.3891 \\
\hline 58 & 1 & landscape & NA & NA & area_mn & $1.04 \mathrm{E}-05$ & 7 & 99.72645 \\
\hline 59 & 1 & landscape & NA & NA & ta & 7.044516 & 7 & 99.72645 \\
\hline 60 & 1 & landscape & NA & NA & area_mn & $1.31 \mathrm{E}-05$ & 8 & 97.71081 \\
\hline 61 & 1 & landscape & NA & NA & ta & 6.902135 & 8 & 97.71081 \\
\hline 62 & 1 & landscape & NA & NA & area_mn & $1.02 \mathrm{E}-05$ & 9 & 99.0357 \\
\hline 63 & 1 & landscape & NA & NA & ta & 6.995723 & 9 & 99.0357 \\
\hline
\end{tabular}


Table 6. Raw results from June 2019

\begin{tabular}{|c|c|c|c|c|c|c|c|c|}
\hline & layer & level & class & id & metric & value & plot_id & percentage_inside \\
\hline 1 & 2 & class & 0 & NA & iji & 57.3288 & 1 & 99.99917 \\
\hline 2 & 2 & class & 1 & NA & $\mathrm{iji}$ & 57.7427 & 1 & 99.99917 \\
\hline 3 & 2 & class & 2 & NA & $\mathrm{iji}$ & 45.94076 & 1 & 99.99917 \\
\hline 4 & 2 & class & 3 & NA & $\mathrm{iji}$ & 59.69857 & 1 & 99.99917 \\
\hline 5 & 2 & landscape & NA & NA & $\mathrm{iji}$ & 60.04741 & 1 & 99.99917 \\
\hline 6 & 2 & class & 0 & NA & $\mathrm{iji}$ & 28.07629 & 2 & 100 \\
\hline 7 & 2 & class & 1 & NA & iji & 53.30547 & 2 & 100 \\
\hline 8 & 2 & class & 2 & NA & $\mathrm{iji}$ & 57.74182 & 2 & 100 \\
\hline 9 & 2 & class & 3 & NA & iji & 40.35966 & 2 & 100 \\
\hline 10 & 2 & landscape & NA & NA & $\mathrm{iji}$ & 38.61139 & 2 & 100 \\
\hline 11 & 2 & class & 0 & NA & $\mathrm{iji}$ & 95.12688 & 3 & 99.76286 \\
\hline 12 & 2 & class & 1 & NA & $\mathrm{iji}$ & 62.88938 & 3 & 99.76286 \\
\hline 13 & 2 & class & 2 & NA & $\mathrm{iji}$ & 2.636491 & 3 & 99.76286 \\
\hline 14 & 2 & class & 3 & NA & $\mathrm{iji}$ & 61.28773 & 3 & 99.76286 \\
\hline 15 & 2 & landscape & NA & NA & $\mathrm{iji}$ & 75.21863 & 3 & 99.76286 \\
\hline 16 & 2 & class & 0 & NA & $\mathrm{iji}$ & 89.80151 & 4 & 98.77784 \\
\hline 17 & 2 & class & 1 & NA & $\mathrm{iji}$ & 61.88414 & 4 & 98.77784 \\
\hline 18 & 2 & class & 2 & NA & $\mathrm{iji}$ & 2.623124 & 4 & 98.77784 \\
\hline 19 & 2 & class & 3 & NA & $\mathrm{iji}$ & 63.06193 & 4 & 98.77784 \\
\hline 20 & 2 & landscape & NA & NA & $\mathrm{iji}$ & 69.84721 & 4 & 98.77784 \\
\hline 21 & 2 & class & 0 & NA & $\mathrm{iji}$ & 98.53067 & 5 & 98.2663 \\
\hline 22 & 2 & class & 1 & NA & $\mathrm{iji}$ & 62.74196 & 5 & 98.2663 \\
\hline 23 & 2 & class & 2 & NA & $\mathrm{iji}$ & 4.69965 & 5 & 98.2663 \\
\hline 24 & 2 & class & 3 & NA & $\mathrm{iji}$ & 62.82436 & 5 & 98.2663 \\
\hline 25 & 2 & landscape & NA & NA & $\mathrm{iji}$ & 77.20106 & 5 & 98.2663 \\
\hline 26 & 2 & class & 0 & NA & $\mathrm{iji}$ & 71.64569 & 6 & 99.95729 \\
\hline 27 & 2 & class & 1 & NA & $\mathrm{iji}$ & 47.65546 & 6 & 99.95729 \\
\hline 28 & 2 & class & 2 & NA & $\mathrm{iji}$ & 0 & 6 & 99.95729 \\
\hline 29 & 2 & class & 3 & NA & $\mathrm{iji}$ & 52.98343 & 6 & 99.95729 \\
\hline 30 & 2 & landscape & NA & NA & $\mathrm{iji}$ & 59.99697 & 6 & 99.95729 \\
\hline 31 & 2 & class & 0 & NA & $\mathrm{iji}$ & 81.76801 & 7 & 99.9326 \\
\hline 32 & 2 & class & 1 & NA & $\mathrm{iji}$ & 43.58816 & 7 & 99.9326 \\
\hline 33 & 2 & class & 2 & NA & $\mathrm{iji}$ & 0.123945 & 7 & 99.9326 \\
\hline 34 & 2 & class & 3 & NA & $\mathrm{iji}$ & 42.85943 & 7 & 99.9326 \\
\hline 35 & 2 & landscape & NA & NA & $\mathrm{iji}$ & 62.81481 & 7 & 99.9326 \\
\hline 36 & 2 & class & 0 & NA & $\mathrm{iji}$ & 94.78344 & 8 & 99.96322 \\
\hline 37 & 2 & class & 1 & NA & $\mathrm{iji}$ & 46.46075 & 8 & 99.96322 \\
\hline 38 & 2 & class & 2 & NA & $\mathrm{iji}$ & 0.007187 & 8 & 99.96322 \\
\hline 39 & 2 & class & 3 & NA & $\mathrm{iji}$ & 46.81544 & 8 & 99.96322 \\
\hline 40 & 2 & landscape & NA & NA & $\mathrm{iji}$ & 70.28042 & 8 & 99.96322 \\
\hline 41 & 2 & class & 0 & NA & $\mathrm{iji}$ & 89.64284 & 9 & 99.96795 \\
\hline 42 & 2 & class & 1 & NA & $\mathrm{iji}$ & 47.96221 & 9 & 99.96795 \\
\hline
\end{tabular}




\begin{tabular}{c|cccccccc}
43 & 2 & class & 2 & NA & iji & 0.028385 & 9 & 99.96795 \\
44 & 2 & class & 3 & NA & iji & 43.99011 & 9 & 99.96795 \\
45 & 2 & landscape & NA & NA & iji & 67.58713 & 9 & 99.96795 \\
46 & 2 & landscape & NA & NA & area_mn & $7.45 E-05$ & 1 & 99.99917 \\
47 & 2 & landscape & NA & NA & ta & 7.06378 & 1 & 99.99917 \\
48 & 2 & landscape & NA & NA & area_mn & $5.48 E-05$ & 2 & 100 \\
49 & 2 & landscape & NA & NA & ta & 7.063842 & 2 & 100 \\
50 & 2 & landscape & NA & NA & area_mn & $3.19 E-05$ & 3 & 99.76286 \\
51 & 2 & landscape & NA & NA & ta & 7.047088 & 3 & 99.76286 \\
52 & 2 & landscape & NA & NA & area_mn & $3.33 E-05$ & 4 & 98.77784 \\
53 & 2 & landscape & NA & NA & ta & 6.977507 & 4 & 98.77784 \\
54 & 2 & landscape & NA & NA & area_mn & $6.99 E-05$ & 5 & 98.2663 \\
55 & 2 & landscape & NA & NA & ta & 6.941373 & 5 & 98.2663 \\
56 & 2 & landscape & NA & NA & area_mn & $1.16 E-05$ & 6 & 99.95729 \\
57 & 2 & landscape & NA & NA & ta & 7.060822 & 6 & 99.95729 \\
58 & 2 & landscape & NA & NA & area_mn & $9.34 \mathrm{E}-06$ & 7 & 99.9326 \\
59 & 2 & landscape & NA & NA & ta & 7.059078 & 7 & 99.9326 \\
60 & 2 & landscape & NA & NA & area_mn & $1.07 E-05$ & 8 & 99.96322 \\
61 & 2 & landscape & NA & NA & ta & 7.061241 & 8 & 99.96322 \\
62 & 2 & landscape & NA & NA & area_mn & $9.21 E-06$ & 9 & 99.96795 \\
63 & 2 & landscape & NA & NA & ta & 7.061575 & 9 & 99.96795
\end{tabular}




\section{Appendix B. R-Script Using Landscapemetrics Library}

\#This script is roughly based on a R-script provided by the National Audubon Society as \#a reference.

\#This is code interspersion and habitat analysis using bird survey points.

\#It takes two raster layers containing four landcover classes each and one survey point \#feature class from a geodatabase

\#Load libraries

library(raster)

library(landscapemetrics) \#This is the main analysis library

library(arcgisbinding)

library(gdalUtils)

library(sp)

\#dyplr library is used for dataframe and table manipulation, in this case combining all \#sample results into one cohesive table per input raster layer

library(dplyr)

arc.check_product()

\# Set the working directory

setwd ("C:\\Project Datal|R\\R_Interspersion\\Classified_TIFF")

\# These Raster layers have four classes each:

\# Open Water, Exposed Mud, Floating Vegetation, and Emergent Vegetation

R1 = raster("Analysis_Input_20181.tif")

R2 = raster("Analysis_Input_June_20191.tif")

\#\#\#Load the Point Layer 
\# Converted from ArcGIS point feature into SpatialPoints and will used in the interspersion sample calculations.

\# Select and open point layer into temporary variable name

\# Extract the shape and associated coordinates

B <- arc.select(arc.open("C:I\Project Datal\Audubon_MIP.gdb\\oints"))

$\mathrm{x}<$-arc.shape $(\mathrm{B})$

geom <- arc.shape $2 \mathrm{sp}(\mathrm{x})$

points_sp <-geom

\#\# This is the Coordinates check, take out or comment out if not needed. \#Convert point data to match coordinates in raster layers for the samle_lsm()

\#pt<- spTransform(points_sp, CRS("+proj=utm +zone=17 +ellps=GRS80

\#+datum=NAD83 +units=m +no_defs"))

\#\#\#\# Analysis Section

\# Use the dyplr library to combine multiple metric results to one tibble dataframe

\#\#Description of the fallowing metrics

\#lsm_1_iji = landscape level interspersion and juxtaposition metric

\#lsm_c_iji = class level interspersion and juxtaposition metric

\#lsm_1_area_mn = mean of patch area (Area and edge metric) is useful paired with the following:

\#lsm_1_ta $=$ total landscape area to give an idea of patch structure

$$
\begin{aligned}
& \text { samples = dplyr }: \text { :bind_rows }( \\
& \text { sample_lsm }(\mathrm{R} 1, \mathrm{y}=\text { points_sp, } \\
& \text { size }=150, \text { \#Set radius in meters } \\
& \text { what }=\mathrm{c}(\text { "lsm_c_iji","lsm_1_iji"), } \\
& \text { shape }=\text { "circle"), }
\end{aligned}
$$


sample_lsm(R1, y = points_sp,

size $=150, \#$ Set radius in meters

what = c("1sm_1_area_mn", "lsm_1_ta"),

shape $=$ "circle",))

\# Repeat for second classification layer

samples $2=$ dplyr ::bind_rows(

sample_lsm(R2, y= points_sp,

size $=150$,

what =c("lsm_c_iji","lsm_1_iji"),

shape $=$ "circle"),

sample_lsm(R2, y = points_sp,

size $=150$,

what = c("1sm_1_area_mn", "1sm_1_ta"),

shape $=$ "circle",))

\#Write results to a CSV for 2018

metrics <- rbind(samples) \#appends metrics to csv

print("csv appended")

write.csv(metrics, paste0("Interspersion_2018.csv"))

print("Done with 2018")

\#Write results to a CSV for June 2019

metrics <- rbind(samples2) \#appends metrics to csv

print("csv appended")

write.csv(metrics, paste0("Interspersion_June_2019.csv"))

print("Done with June 2019")

sessionInfo() 


\section{Appendix C. Additional Class Level Charts}

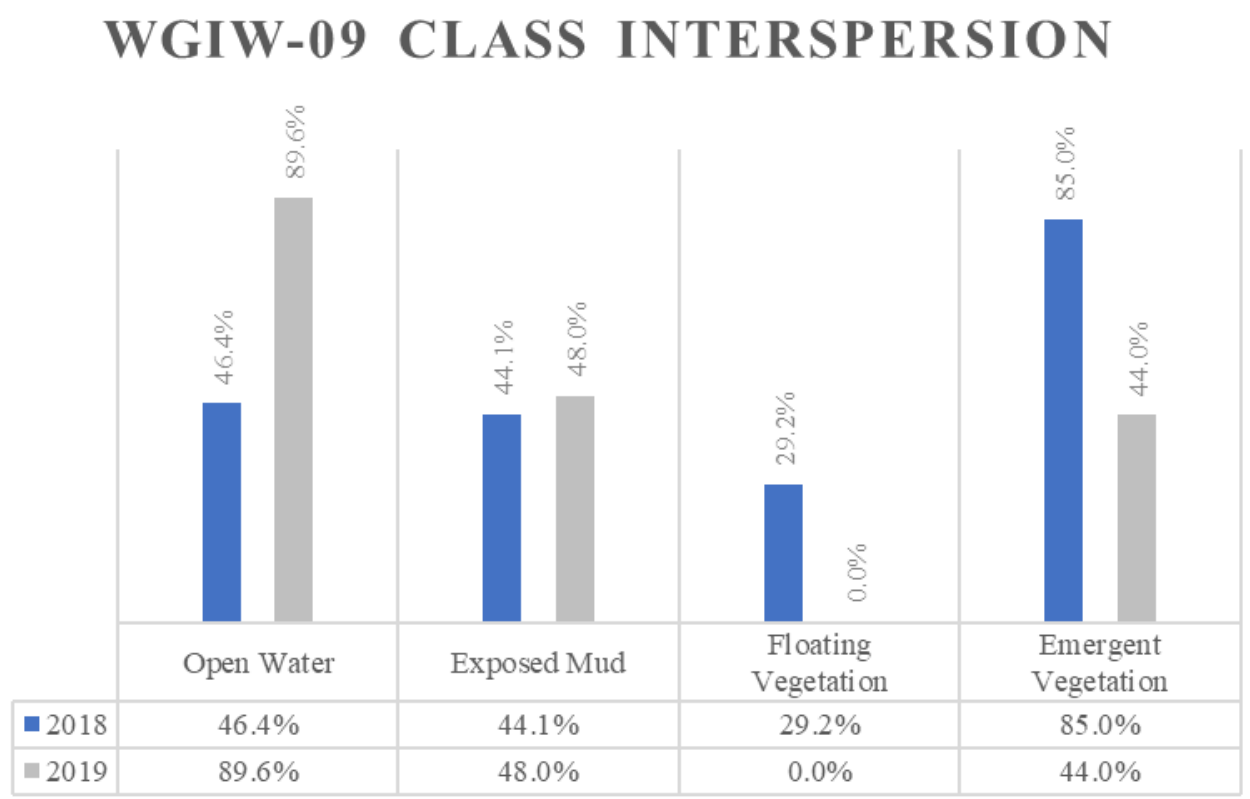

Figure C-1: Study Area 9 Class Interspersion 


\section{WIGW-05 CLASS INTERSPERSION}

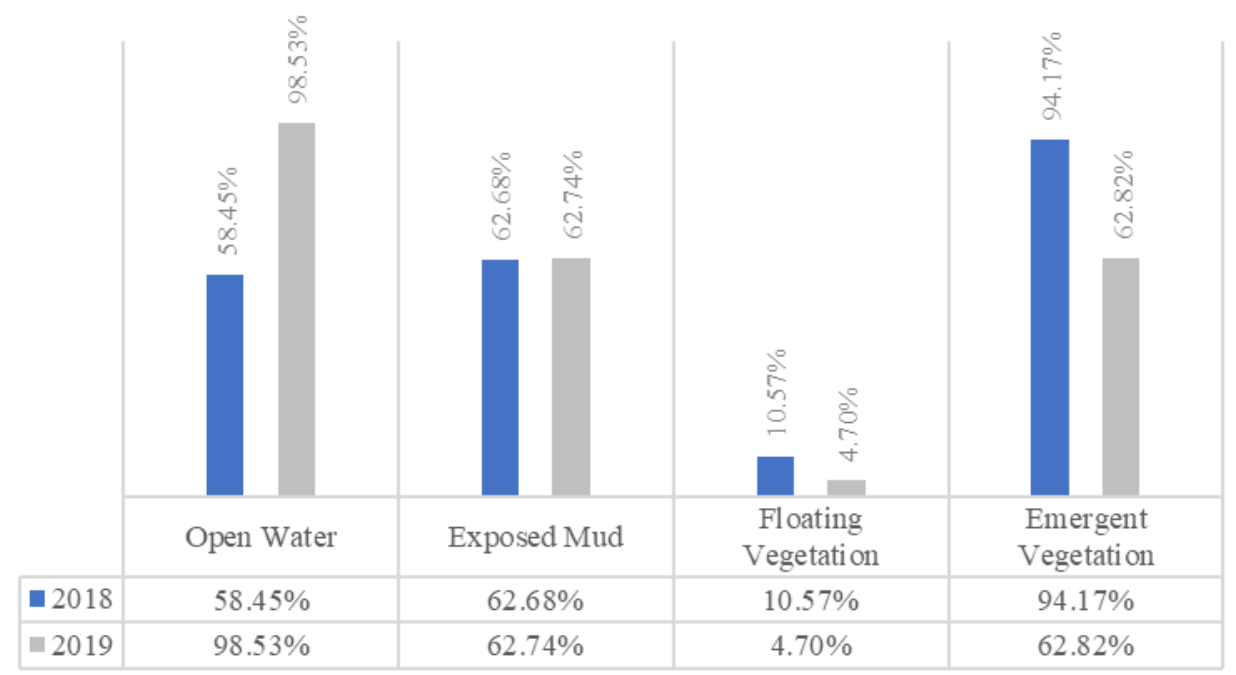

Figure C-2: Study Area 5 Class Interspersion

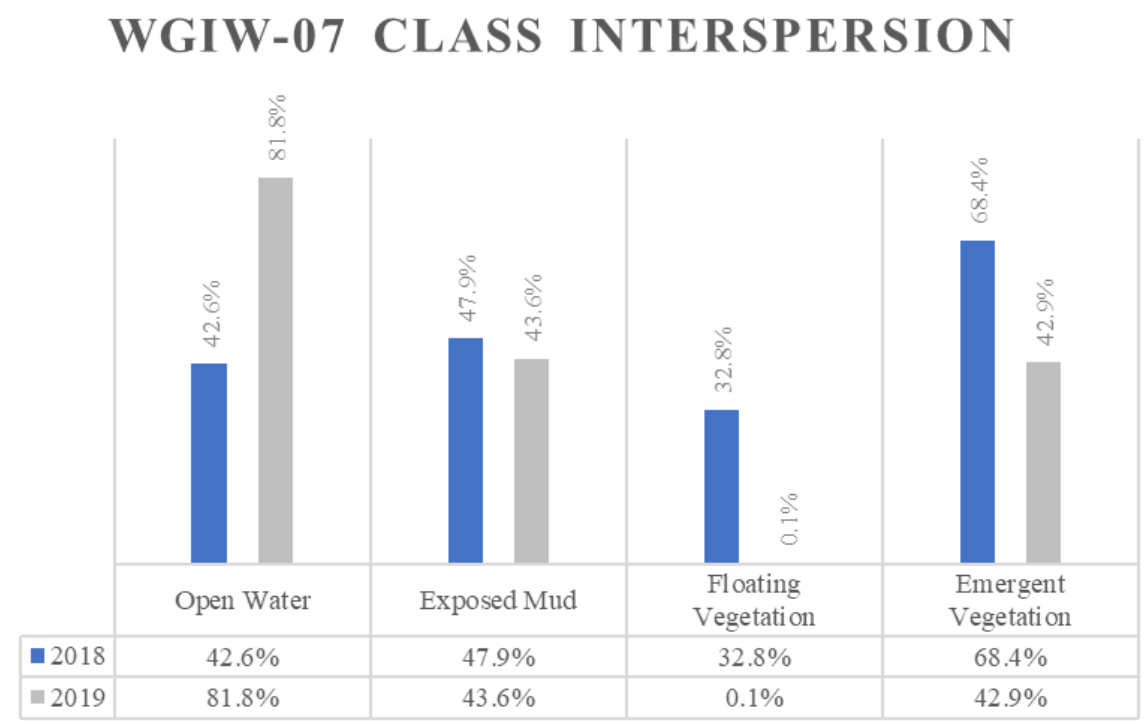

Figure C-3: Study Area 7 Class Interspersion 


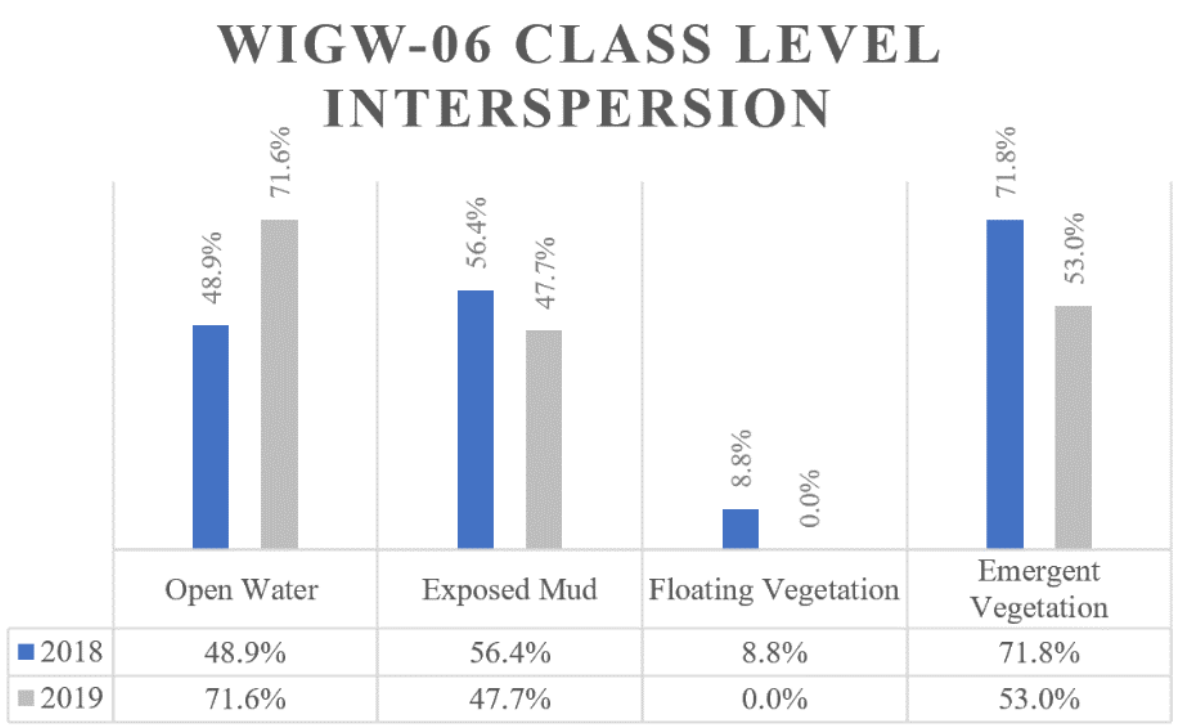

Figure C-4: Study Area 6 Class Interspersion

\section{WIGW-03 CLASS INTERSPERSION}

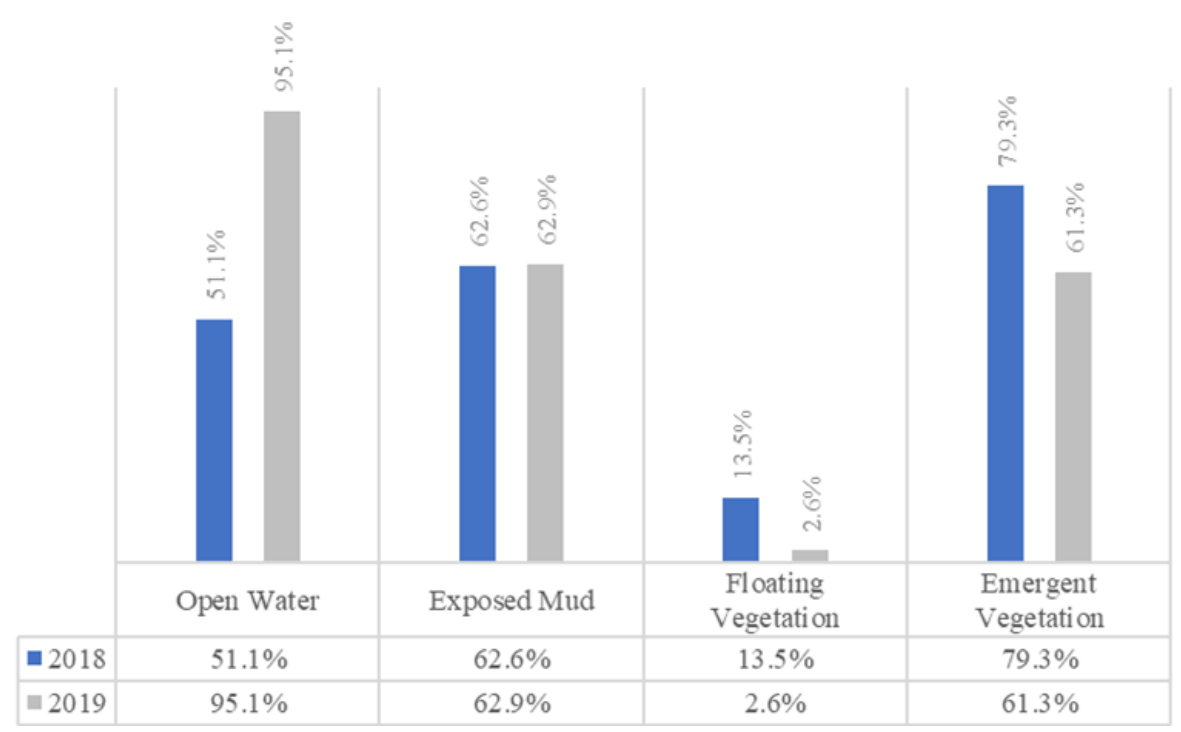

Figure C-5: Study Area 3 Class Interspersion 


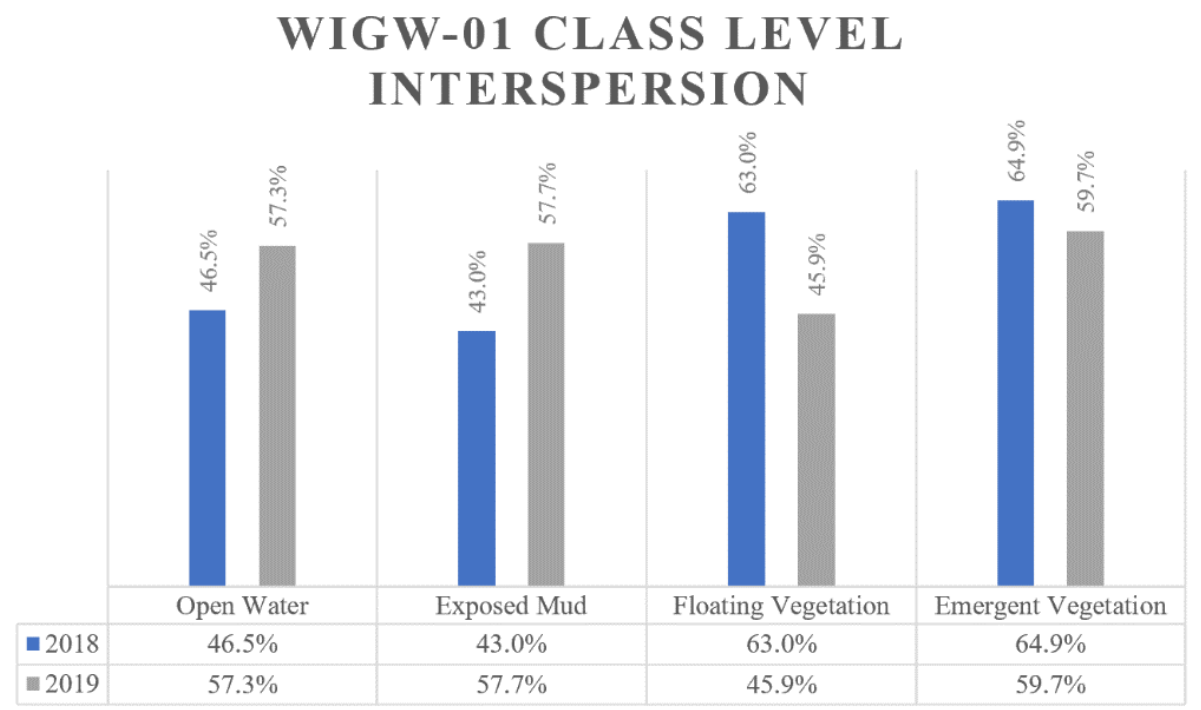

Figure C-6: Study Point 1 Class Interspersion 